\title{
Centrality Rewarding Shapley and Myerson Values for Undirected Graph Games
}

\author{
Anna Khmelnitskayal \\ Gerard van der Laan² \\ Dolf Talman ${ }^{3}$
}

2 Faculty of Economics and Business Administration, VU University Amsterdam, the Netherlands;

3 Tilburg University, the Netherlands. 
Tinbergen Institute is the graduate school and research institute in economics of Erasmus University Rotterdam, the University of Amsterdam and VU University Amsterdam.

More TI discussion papers can be downloaded at http://www.tinbergen.nl

Tinbergen Institute has two locations:

Tinbergen Institute Amsterdam

Gustav Mahlerplein 117

1082 MS Amsterdam

The Netherlands

Tel.: +31(0)20525 1600

Tinbergen Institute Rotterdam

Burg. Oudlaan 50

3062 PA Rotterdam

The Netherlands

Tel.: +31(0)10 4088900

Fax: +31(0)10 4089031 


\title{
Centrality rewarding Shapley and Myerson values for undirected graph games
}

\author{
Anna Khmelnitskaya* Gerard van der Laan $^{\dagger}$ \\ Dolf Talman
}

September 2, 2016

\begin{abstract}
In this paper we introduce two values for cooperative games with communication graph structure. For cooperative games the shapley value distributes the worth of the grand coalition amongst the players by taking into account the worths that can be obtained by any coalition of players, but does not take into account the role of the players when communication between players is restricted. Existing values for communication graph games as the Myerson value and the average tree solution only consider the worths of connected coalitions and respect only in this way the communication restrictions. They do not take into account the position of a player in the graph in the sense that, when the graph is connected, in the unanimity game on the grand coalition all players are treated equally and so players with a more central position in the graph get the same payoff as players that are not central. The two new values take into account the position of a player in the graph. The first one respects centrality, but not the communication abilities of any player. The second value reflects both centrality and the communication ability of each player. That implies that in unanimity games players that do not generate worth but are needed to connect worth generating players are treated as those latter players, and simultaneously players that are more central in the graph get bigger shares in the worth than players that are less central. For both values an axiomatic characterization is given on the class of connected cycle-free graph games.
\end{abstract}

Keywords: cooperative game, Shapley value, communication graph, restricted

${ }^{*}$ A.B. Khmelnitskaya, Saint-Petersburg State University, Faculty of Applied Mathematics, Universitetskii prospekt 35, 198504, Petergof, SPb, Russia, e-mail: a.b.khmelnitskaya@utwente.nl. The research of Anna Khmelnitskaya was supported by RFBR (Russian Foundation for Basic Research) grant \#16-01-00713 and NWO (Dutch Organization of Scientific Research) grant \#040.11.516. Her research was done partially during her stay at Vrije Universiteit Amsterdam and the University of Twente, the hospitality of both universities is highly appreciated.

${ }^{\dagger}$ G. van der Laan, Department of Econometrics and Tinbergen Institute, Vrije Universiteit Amsterdam, De Boelelaan 1105, 1081 HV Amsterdam, The Netherlands, e-mail: g.vander.laan@vu.nl

${ }_{\ddagger}^{\ddagger}$ A.J.J. Talman, CentER, Department of Econometrics \& Operations Research, Tilburg University, P.O. Box 90153, 5000 LE Tilburg, The Netherlands, e-mail: talman@tilburguniversity.edu 
cooperation, centrality.

\section{JEL Classification Number: C71}

\section{Introduction}

In classical cooperative game theory it is assumed that any coalition of players may form. However, in many practical situations the set of feasible coalitions is restricted by some social, economical, hierarchical, or technical structure. The study of TU games with limited cooperation introduced by means of undirected communication graphs, later on for brevity called graph games, was initiated by Myerson [?]. Assuming that only connected players can cooperate, the Myerson value for graph games is defined as the Shapley value [?] of the so-called restricted game in which the worth of each coalition is equal to the sum of the worths of its components in the graph.

Following Myerson, several other solutions for graph games have been proposed. Since the Shapley value takes the average of the marginal vectors of the game over all linear orderings on the set of players, the Myerson value takes the average of all marginal vectors of the Myerson restricted game. Bilbao (2003) introduced the Shapley value for games under augmenting systems. Such systems yield restrictions on the cooperation opportunities generalizing the restrictions imposed by a graph. Applied to graph games the solution proposed in Bilbao (2003) takes the average of the marginal vectors over only those linear orderings that induce sequences of connected coalitions. Solutions using the notion of (rooted) trees (partial orderings) instead of linear orderings tree were introduced by Herings, van der Laan, Talman and Yang (2010) and Koshevoy and Talman (2014). For a given connected graph, a spanning tree of the graph is a connected cycle-free subgraph, whereas a tree is admissible if it is a connected cycle-free graph and in the tree every node is linked to precisely one node in each component of the subgraph on the set of his subordinates in the tree. The average tree solution for graph games introduced in Herings et al. (2010) assigns to each player the average of the player's marginal contributions to his subordinates in the Myerson restricted game over all admissible spanning trees. The gravity center restricted to graph games, introduced by Koshevoy and Talman (2014) for games with restricted communication represented by an arbitrary collection of feasible coalitions, assigns to each player the average of the player's marginal contributions to his subordinates in the Myerson restricted game over all admissible trees, not necessarily spanning trees.

The four solutions mentioned above suffer from some drawbacks, which we illustrate here with an example. Let the graph be given by a star. We call the center of the star the hub and all other players (nodes) the satellites of the star. Further, let the game be the unanimity game on the set of all satellites, i.e. only the coalition of all players except the hub generates worth. Note that the hub is needed to connect the satellites, so the players of the worth generating coalition can not communicate without the hub. The Myerson value and the average tree value take into account that the hub is needed for communication. In this example both values distribute the worth equally amongst all players. However, these values don't take into account the central position of the hub. The Shapley value of Bilbao has the null player property. For this example it assigns the 
same outcome as the Shapley value, leaving the hub with zero payoff. It therefore does neither reward communication, nor centrality. The gravity center gives equal payoffs to all satellites, while the hub gets much less, it recognizes to some extent communication, but not centrality.

In general, a communication graph typically introduces asymmetries between the players with respect to their communication abilities and so dividing the worth equally in a unanimity game is not a priori reasonable. On the contrary, it seems more natural that a player with more connections is rewarded better. Considering the unanimity game on the grand coalition, for any graph structure the Myerson value assigns equal payoffs to the players. Also the average tree solution is doing so for every cycle-free graph. So, these solutions do not reward centrality. When the graph is a star, all four solutions give the same outcome in case of the unanimity game on the grand coalition as in case of the unanimity game on all satellites. So, also when the hub is needed not only for communication but also for generating worth, the gravity center gives less payoff to the hub than to the satellites and the Bilbao value even gives zero payoff to the center. We can say that in the latter two values centrality is punished.

Values that take centrality into account are the position value introduced in Borm, Owen, and Tijs (1992) and the Harsanyi power solutions given in van den Brink, van der Laan, and Pruzhanski (2011). Drawbacks of these solutions are discussed in Section 6. In this paper we introduce two new solution concepts. The first one rewards centrality, but does not take into account the communication ability of players. In particular, it has the well-known null player property, and so any null player in the game gets zero payoff, even if this player is needed to connect non-null players. The second new value rewards both communication and centrality. Similar to the Shapley value of Bilbao (2003) the two values consider a subset of the collection of all linear orderings of the players. However, while the value of Bilbao considers the linear orderings that satisfy the condition that for every player the set of players ranked lower in the ordering is connected in the graph, we now do the opposite, namely we consider the linear orderings that satisfy the condition that for every player the set of higher-ordered players is connected in the graph. In the usual interpretation a linear ordering describes the order in which players form the grand coalition, starting with a single player. Actually, in this new setting we can interpret a linear ordering as the order in which players leave subsequently the grand coalition in such way that after every departure the set of remaining players is still connected in the graph. The new values are then obtained by taking the average over all these orderings of the marginal vectors with respect to the game itself and the Myerson restricted game, respectively. We call the first one the centrality rewarding Shapley value and the second one centrality rewarding Myerson value. Contrary to the Shapley value of Bilbao, the two values reward players that have a central position in the graph. In case of the unanimity game on the grand coalition both solutions yield the same outcome. In this case the values have the property that the more central the position of a player is, the higher is the player's payoff. In particular, when the graph is a star, both values assign half of the worth of the grand coalition to the hub, while the other half is shared equally by the satellites. More generally, when applied to the unanimity game on the grand coalition the new values induce a centrality measure for undirected graphs, see Khmelnitskaya, van der Laan, and Talman (2016).

In the classical interpretation of a linear ordering the players enter a room one by 
one aiming to create the grand coalition, and every player is rewarded by his marginal contribution to the players who entered before. In this interpretation in each ordering the first player gets only his own worth, while the last player gets its marginal contribution to the grand coalition. However, then the grand coalition up to him is already formed, and so, his role in creating the grand coalition is minimal. In real life it is typically the other way around. The person that starts some group activity expects to get as payoff his marginal contribution in the creation of the grand coalition, not just his own worth. This leads to our new interpretation. It starts with the grand coalition and the first player that leaves receives its own payoff. When there are no restrictions on cooperation, all coalitions of players are feasible and, therefore, all orderings of players are admissible, so that both interpretations result in the same outcome, the Shapley value. In case of a communication graph game only connected coalitions are considered and then the two interpretations lead to different solution concepts. While the Shapley value of Bilbao inherits the distribution model of the classical interpretation of the Shapley value, our new values follow the distribution model of the alternative interpretation.

The structure of the paper is as follows. Section 2 contains preliminaries and in Section 3 we discuss the four existing values for communication graph games mentioned above. In Section 4 we introduce the two new values and in Section 5 we provide an axiomatic characterization of the values on the class of connected cycle-free graph games. Further remarks are made in Section 6.

\section{Preliminaries}

A cooperative game with transferable utility ( $T U$ game) is a pair $(N, v)$, where $N=$ $\{1, \ldots, n\}$ is a finite set of $n \geq 2$ players and $v: 2^{N} \rightarrow \mathbb{R}$ is a characteristic function with $v(\emptyset)=0$, assigning to any coalition $S \subseteq N$ its worth $v(S)$ that can be freely distibuted among the members of $S$. The set of TU games with fixed player set $N$ is denoted $\mathcal{G}_{N}$. For simplicity of notation and if no ambiguity appears we write $v$ when we refer to a game $(N, v)$. For a subset $\mathcal{G} \subseteq \mathcal{G}_{N}$, a value on $\mathcal{G}$ is a function $f: \mathcal{G} \rightarrow \mathbb{R}^{N}$ that assigns to every $v \in \mathcal{G}$ a vector $f(v) \in \mathbb{R}^{N}$ where $f_{i}(v)$ is the payoff to player $i \in N$ in the TU game $v$. In the sequel we denote the cardinality of a finite set $A$ by $|A|$. For $S \subset N$ and $i \in N \backslash S$ we sometimes denote $S \cup\{i\}$ by $S_{+i}$.

For $T \subseteq N$ the unanimity game $u_{T}$ is defined as $u_{T}(S)=1$ if $T \subseteq S$, and $u_{T}(S)=0$ otherwise. For a unanimity game $u_{T}, T$ is called the unanimity coalition. It is well known (Shapley, 1953) that $\left\{u_{T} \mid T \in 2^{N}, T \neq \emptyset\right\}$ forms a basis in $\mathcal{G}_{N}$, i.e., for every $\mathrm{TU}$ game $v \in \mathcal{G}_{N}$ its characteristic function $v$ can be uniquely represented in the linear form as

$v=\sum_{T \subseteq N, T \neq \emptyset} \lambda_{T}(v) u_{T}$, where $\lambda_{T}(v)=\sum_{S \subseteq T}(-1)^{|T|-|S|} v(S)$, for all $T \subseteq N, T \neq \emptyset$. By the

Möbius transform we have that $v(S)=\sum_{T \subseteq S} \lambda_{T}(v)$ for all $S \subseteq N$. Following Harsanyi (1959) the coefficient $\lambda_{v}(T)$ is referred to as the dividend of the coalition $T$ in game $v$ and has the natural interpretation as the extra revenue from cooperation among its players that they did not already realize by cooperating in all proper subcoalitions of $S$.

For a linear ordering $\pi: N \rightarrow N$ and $i \in N, \pi(i)$ is the position of player $i$ in $\pi$. The set of linear orderings on $N$ is denoted $\Pi(N)$. For $\pi \in \Pi(N)$ and $i \in N$ we denote 
$P^{\pi}(i)=\{j \in N \mid \pi(j)<\pi(i)\}$ as the set of players with position in $\pi$ before $i$, and we denote $\bar{P}^{\pi}(i)=P^{\pi}(i) \cup\{i\}$. For TU game $v \in \mathcal{G}_{N}$ and linear ordering $\pi \in \Pi(N)$, the marginal vector $m^{\pi}(v) \in \mathbb{R}^{N}$ is given by $m_{i}^{\pi}(v)=v\left(\bar{P}^{\pi}(i)\right)-v\left(P^{\pi}(i)\right), i \in N$. The Shapley value on the class of TU games is the function $S h$ that assigns to every $v \in \mathcal{G}_{N}$ the vector $S h(v) \in \mathbb{R}^{N}$ given by

$$
S h(v)=\frac{1}{n !} \sum_{\pi \in \Pi(N)} m^{\pi}(v) .
$$

An (undirected) graph on $N$ consists of $N$ as its set of nodes (players) and a collection $\Gamma \subseteq\{\{i, j\} \subseteq N \mid i \neq j\}$ of unordered pairs of distinct elements of $N$. The elements of $\Gamma$ are called links or edges. The collection of undirected graphs on $N$ is denoted by $\Gamma_{N}$ and we refer to an element on this class by its set of edges $\Gamma$. Given a graph $\Gamma \in \Gamma_{N}$ and $S \in 2^{N} \backslash\{\emptyset\}$, the graph $\left.\Gamma\right|_{S}$ with $\left.\Gamma\right|_{S}=\{\{i, j\} \in \Gamma \mid i, j \in S\}$ is the subgraph of $\Gamma$ on $S$. Note that $\left.\Gamma\right|_{N}=\Gamma$.

Let $\Gamma \in \Gamma_{N}$ be a graph on $N$. A sequence of $k$ distinct nodes $\left(i_{1}, \ldots, i_{k}\right)$ is a path in $\left.\Gamma\right|_{S}$ if $\left.\left\{i_{\ell}, i_{\ell+1}\right\} \in \Gamma\right|_{S}$ for $\ell=1, \ldots, k-1$. Two nodes $i, j \in N$ are connected in $\left.\Gamma\right|_{S}$ if there is a path $\left(i_{1}, \ldots, i_{k}\right)$ in $\left.\Gamma\right|_{S}$ with $i_{1}=i$ and $i_{k}=j$. A subgraph $\left.\Gamma\right|_{S}$ is connected, or shortly coalition $S$ is connected, if every two nodes in $S$ are connected in $\left.\Gamma\right|_{S}$. A coalition $K \subseteq S$ is a component of $\left.\Gamma\right|_{S}$, or $S$, if $K$ is a maximal connected subset of $S$, i.e., $K$ is connected and for every $i \in S \backslash K$ the set $K \cup\{i\}$ is not connected in $\Gamma$. The set of components of $\left.\Gamma\right|_{S}$ is denoted by $S / \Gamma$. $\Gamma$ is cycle-free if for every two different nodes $i$ and $j$ either $i$ and $j$ are not connected or there is precisely one path in $\Gamma$ connecting $i$ and $j$. Note that $\Gamma \in \Gamma_{N}$ has precisely $|N|-1$ links when $\Gamma$ is connected and cycle-free. The collection of connected graphs in $\Gamma_{N}$ is denoted by by $\Gamma_{N}^{c}$, and the collection of connected cycle-free graphs by $\Gamma_{N}^{c f}$. Node $i \in N$ is an extreme node of graph $\Gamma \in \Gamma_{N}^{c}$ when $N \backslash\{i\}$ is connected. A connected cycle-free graph is a linear graph if each of its nodes has at most two neighbors, and it is a star if there is one node, called the hub, and any other node, called a satellite, is only connected to the hub.

A directed graph, or digraph, on $N$ consists of $N$ as its set of nodes and a collection $D \subseteq\{(i, j) \mid i, j \in N, i \neq j\}$ of ordered pairs of distinct elements of $N$. The elements of $D$ are called arcs. We shortly refer to a digraph by its collection of $\operatorname{arcs} D$. For a digraph $D$, a sequence of distinct nodes $\left(i_{1}, \ldots, i_{r}\right), r \geq 2$, is a directed path from $i_{1}$ to $i_{r}$ if $\left(i_{h}, i_{h+1}\right) \in D$ for $h=1, \ldots, r-1$. Node $j$ is a successor of node $i$ (and $i$ a predecessor of $j$ ) if $(i, j) \in D$, and $j$ is a subordinate of $i$ (and $i$ a superior of $j$ ) if there is a directed path from $i$ to $j$ in $D$. For $i \in N, S_{D}(i)$ is the set of successors of $i, \bar{S}_{D}(i)$ the set of subordinates of $i$, and $\widehat{S}_{D}(i)=\bar{S}_{D}(i) \cup\{i\}$. Note that $\bar{S}_{D}(i)=\cup_{j \in S_{D}(i)} \widehat{S}_{D}(j)$ for all $i \in N$.

A digraph $D$ on $N$ is a (rooted) tree if there is a unique node without predecessors, the root of the tree, denoted $r(D)$, and for every other node in $N$ there is a unique directed path in $D$ from the root to that node. Following Demange (2004), for a tree $D$ and $i \in N$, the set $\widehat{S}_{D}(i)$ consisting of $i$ and its subordinates is called the team of $i$. Note that $\widehat{S}_{D}(r(D))=N$ and $\widehat{S}_{D}(i)=\{i\}$ when $i$ has no successors. A node without successors is called a leaf.

For given $\Gamma \in \Gamma_{N}^{c}$ a tree $D$ is admissible for $\Gamma$ if for each $(i, j) \in D$ it holds that $\widehat{S}_{D}(j)$ is a component in the subgraph $\left.\Gamma\right|_{\bar{S}_{D}(i)}$ of $(N, \Gamma)$, i.e., when $(i, j) \in D$ then the team of 
$j$ is a component of the subgraph of $\Gamma$ on the set of subordinates of $i$. A tree $D$ is a spanning tree of $\Gamma$ if $\{i, j\} \in \Gamma$ when $(i, j) \in D$. Note that when $\Gamma$ is cycle-free, then for each $i \in N$ there is precisely one spanning tree with node $i$ as root. Given $\Gamma \in \Gamma_{N}^{c}, T^{a}(\Gamma)$ denotes the collection of admissible trees and $T^{s}(\Gamma)$ denotes the collection of admissible spanning trees.

\section{Values for games with graph structure}

In this paper the node set $N$ of a graph is a set of players and the graph represents a communication structure on the set of players. The players of a coalition $S$ can communicate amongst each other if $S$ is connected in $\Gamma$. A TU game with communication structure, shortly graph game, with player set $N$ is given by the pair $(v, \Gamma)$ with $v \in \mathcal{G}_{N}$ and $\Gamma \in \Gamma_{N}$. We denote the collection of graph games on fixed player set $N$ by $\mathcal{G}_{N}^{\Gamma}$. The subclass of graph games $(v, \Gamma)$ on $N$ with $\Gamma \in \Gamma_{N}^{c}$ is denoted by $\mathcal{G}_{N}^{\Gamma_{c}}$ and the subclass with $\Gamma \in \Gamma_{N}^{c f}$ is denoted by $\mathcal{G}_{N}^{\Gamma_{c f}}$. A solution $f$ on a subset $\mathcal{G} \subseteq \mathcal{G}_{N}^{\Gamma}$ assigns a unique payoff vector $f(v, \Gamma) \in \mathbb{R}^{N}$ to every $(v, \Gamma) \in \mathcal{G}$.

For a graph game $(v, \Gamma)$, Myerson (1977) assumes that players in a coalition $S \subseteq N$ can only cooperate when they are able to communicate with each other, so when $S$ is connected in $(N, \Gamma)$. For $(v, \Gamma) \in \mathcal{G}_{N}$ the (Myerson) restricted game $v^{\Gamma} \in \mathcal{G}_{N}$, is defined by

$$
v^{\Gamma}(S)=\sum_{T \in S / \Gamma} v(T), \text { for all } S \subseteq N
$$

The Myerson value, denoted by $\mu$, on the class of graph games is defined as the Shapley value of its restricted game,

$$
\mu(v, \Gamma)=S h\left(v^{\Gamma}\right), \text { for every }(v, \Gamma) \in \mathcal{G}_{N}^{\Gamma} .
$$

The Myerson value of a graph game is the average over all linear orderings of the marginal vectors of a $\mathrm{TU}$ game that is obtained from the original game by taking into account the communication restrictions induced by the graph.

In case communication restrictions are induced by augmenting systems, Bilbao (2003) proposes a different approach. Restrictions induced by connected graphs are a special case of restrictions induced by augmenting systems. Instead of using these restrictions to modify the game, in the approach of Bilbao (2003) the communication restrictions limit the collection of linear orderings. When applied to a connected graph $\Gamma$, a linear ordering $\pi \in \Pi(N)$ is consistent with $\Gamma$ when for every $i \in N$ the set $\bar{P}^{\pi}(i)$ is connected in $\Gamma$, i.e., the linear ordering generates a sequence of coalitions that are connected and thus can cooperate. Let $\Pi^{\Gamma}(N) \subseteq \Pi(N)$ be the collection of all linear orderings consistent with $\Gamma$. Then the Shapley value for graph games as introduced in Bilbao, denoted by $S h^{B}$, is defined as

$$
S h^{B}(v, \Gamma)=\frac{1}{\left|\Pi^{\Gamma}(N)\right|} \sum_{\pi \in \Pi^{\Gamma}(N)} m^{\pi}(v) \text {, for every }(v, \Gamma) \in \mathcal{G}_{N}^{\Gamma_{c}} .
$$

This value averages over a subset of marginal vectors of the original game $v$, whereas the Myerson values averages over all marginal vectors of a modified game. Since for 
every $\pi \in \Pi^{\Gamma}(N)$ and every $i \in N$ the set $\bar{P}^{\pi}(i)$ is connected and therefore $v\left(\bar{P}^{\pi}(i)\right)=$ $v^{\Gamma}\left(\bar{P}^{\pi}(i)\right)$, it holds that $S_{h}^{B}(v, \Gamma)=S h^{B}\left(v^{\Gamma}, \Gamma\right)$.

On the class of connected cycle-free graph games, Herings, van der Laan, and Talman (2008) introduces the average tree solution, generalized by Herings et al. (2010) to the class of connected graph games. This solution, denoted by AT, assigns to each $(v, \Gamma) \in \mathcal{G}_{N}^{\Gamma_{c}}$ the average of a collection of payoff vectors. Each vector in this collection is associated with precisely one of the admissible spanning trees, the so-called hierarchical outcome on that tree, as introduced by Demange (2004). Given $(v, \Gamma)$, the hierarchical outcome on an admissible tree $D \in T^{a}(\Gamma)$ is the vector $h^{D}(v, \Gamma) \in \mathbb{R}^{N}$, defined by

$$
h_{j}^{D}(v, \Gamma)=v\left(\widehat{S}_{D}(j)\right)-\sum_{h \in S_{D}(j)} v\left(\widehat{S}_{D}(h)\right), \quad j \in N,
$$

i.e., in the hierarchical outcome $h^{D}(v, \Gamma)$ the payoff of player $j$ is equal to the worth of his team in tree $D$ minus the sum of the worths of the teams of his successors in the tree. Consequently, a leaf $i$ in $D$ gets its own worth $v(\{i\})$ and root $r(D)$ receives the worth of the grand coalition $v(N)$ minus the sum of the worths of the teams of his successors in $D$. The average tree solution, denoted by AT, assigns to each $(v, \Gamma) \in \mathcal{G}_{N}^{\Gamma_{c}}$ the vector $A T(v, \Gamma) \in \mathbb{R}^{N}$ given by

$$
A T(v, \Gamma)=\frac{1}{\left|T^{s}(\Gamma)\right|} \sum_{D \in T^{s}(\Gamma)} h^{D}(v, \Gamma),
$$

i.e., it assigns the average of the hierarchical outcomes on all admissible spanning trees. Note that this is the average over $n=|N|$ hierarchical outcomes when $\Gamma$ is cycle-free.

The gravity center, introduced by Koshevoy and Talman (2014), is a solution on the class of games with restricted cooperation represented by an arbitrary collection of feasible coalitions. On the class of connected graph games the gravity center, denoted by GC, assigns to each $(v, \Gamma) \in \mathcal{G}_{N}^{\Gamma_{c}}$ the vector $G C(v, \Gamma) \in \mathbb{R}^{N}$ given by

$$
G C(v, \Gamma)=\frac{1}{\left|T^{a}(\Gamma)\right|} \sum_{D \in T^{a}(\Gamma)} h^{D}(v, \Gamma),
$$

i.e., it assigns the average of the hierarchical outcomes on all admissible trees.

Note that the Myerson value and the Bilbao value both take the average of marginal vectors over a collection of linear orderings, whereas the average tree solution and the gravity center take the average over a collection of trees (partial orderings).

We now illustrate the four solutions by some examples. The examples show that none of the four solutions reward centrality of players in the graph.

Example 3.1 Take the linear graph with node set $N=\{1,2,3,4\}$, as depicted in Figure 3.1.

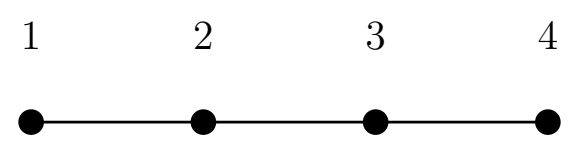

Figure 3.1 
There are eight consistent linear orderings, one with $\pi(1)=1$, given by $\pi=$ $(1,2,3,4)$, and three with $\pi(2)=1$, given by $(2,1,3,4),(2,3,1,4)$, and $(2,3,4,1)$. Similarly, there are three consistent linear orderings with $\pi(3)=1$ and one with $\pi(4)=1$. Note that players 1 and 4 both have the last position in four of the eight consistent linear orderings.

There are four admissible spanning trees, one with each player as root. There are fourteen admissible trees, five with player 1 as root, two with player 2 as root, two with player 3 as root, and five with player 4 as root, as depicted in Figure 3.2.

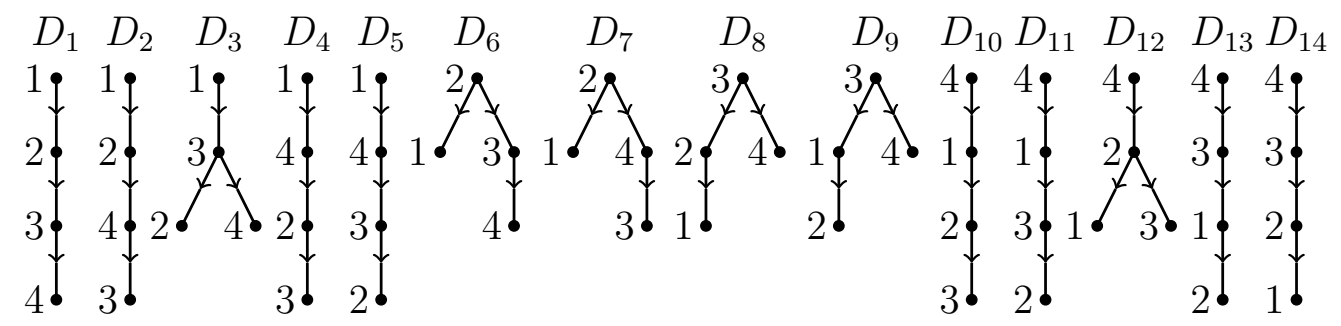

Figure 3.2

We consider unanimity games $u_{N}, u_{124}$ (short for $u_{\{1,2,4\}}$ ), $u_{123}$, and $u_{13}$. Their Myerson restricted games are given by $u_{N}^{\Gamma}=u_{124}^{\Gamma}=u_{N}$ and $u_{123}^{\Gamma}=u_{13}^{\Gamma}=u_{123}$. The payoff vectors for the four solutions are obtained by applying the formulas (??), (??), (??), and (??), respectively. The results are given in Table 3.1.

\begin{tabular}{|c|c|c|c|c|}
\hline$v$ & $\mu$ & $S h^{B}$ & $A T$ & $G C$ \\
\hline$u_{N}$ & $(1 / 4,1 / 4,1 / 4,1 / 4$ & $(1 / 2,0,0,1 / 2)$ & $(1 / 4,1 / 4,1 / 4,1 / 4)$ & $(5 / 14,2 / 14,2 / 14,5 / 14)$ \\
$u_{124}$ & $(1 / 4,1 / 4,1 / 4,1 / 4)$ & $(1 / 2,0,0,1 / 2)$ & $(1 / 4,1 / 4,1 / 4,1 / 4)$ & $(5 / 14,2 / 14,2 / 14,5 / 14)$ \\
$u_{123}$ & $(1 / 3,1 / 3,1 / 3,0)$ & $(3 / 4,0,1 / 4,0)$ & $(1 / 4,1 / 4,1 / 2,0)$ & $(7 / 14,3 / 14,4 / 14,0)$ \\
$u_{13}$ & $(1 / 3,1 / 3,1 / 3,0)$ & $(3 / 4,0,1 / 4,0)$ & $(1 / 4,1 / 4,1 / 2,0)$ & $(7 / 14,3 / 14,4 / 14,0)$ \\
\hline
\end{tabular}

\section{Table 3.1}

Table 3.1 shows that all four solutions give the same outcome for the two characteristic functions $u_{N}$ and $u_{124}$ and for the two characteristic functions $u_{123}$ and $u_{13}$. For the Myerson, AT and GC solutions this follows from the fact that $u_{124}^{\Gamma}=u_{N}^{\Gamma}=u_{N}$ and $u_{13}^{\Gamma}=u_{123}^{\Gamma}=u_{123}$. For $S h^{B}$ it holds that for $u_{N}$ only the two extreme players 1 and 4 in $(N, \Gamma)$ get a positive payoff, and for $u_{123}$ only the two extreme players 1 and 3 in the subgraph $\left.\Gamma\right|_{\{1,2,3\}}$. Therefore the payoffs do not change when a non-extreme player is not needed to generate worth.

For the characteristic functions $u_{N}$ and $u_{123}$, the Myerson value treats all players in the unanimity set equally, and also all players in $N$ and $\{1,2,3\}$ are treated equally for $u_{124}$ and $u_{13}$. This means that players that are not in a unanimity set are rewarded when they are needed for the communication between the players in the unanimity set. On the other hand, the equal treatment implies that players are not rewarded for centrality. In the case $T=N$ the two extreme players 1 and 4 in $(N, \Gamma)$ get the same payoff as the 
two non-extreme players 2 and 3 . Also in case $T=\{1,2,3\}$ the two extreme players 1 and 3 in the subgraph $\left.\Gamma\right|_{T}$ get the same payoff as the central player 2 in this subgraph.

Also the average tree solution rewards players that are needed for communication, but again players are not rewarded for centrality. In case of the unanimity coalitions $T=\{1,2,3\}$ and $T=\{1,3\}$, players 1 and 2 get the same payoff. However, here we see that an extreme player is rewarded for being needed to reach players outside the unanimity coalition. In fact, in both cases player 3 gets twice as much as the players 1 and 2. In this interpretation player 3 also represents player 4 and gets a share for the player that he represents, for more details see Herings et al. (2008) and Mishra and Talman (2010).

The solution of Bilbao does not reward for communication, because every player not in the unanimity coalition gets zero payoff. However, not only a player is not rewarded for communication, but a player is even punished for centrality. As noted above, only the extreme players of the unanimity coalition get non-zero payoff. Also in unanimity game $u_{123}$, player 1 gets three times as much as player 3 . So, not only player 2 is punished for its central position, but compared to player 1 also player 3 is punished for being needed to be connected with the outside player 4. This seems to make the solution of Bilbao unreasonable.

The gravity center rewards for communication, but suffers from the same drawbacks as the Bilbao solution from the viewpoint of centrality. For the unanimity games $u_{N}$ and $u_{124}$ the players 2 and 3 are treated equally, but they get less payoff than extreme players 1 and 4 . For the unanimity games $u_{123}$ (and also $u_{13}$ ) player 2 gets a positive payoff, but less than the players 1 and 3. Moreover, player 3 gets less payoff than player 1. So, not only player 2 is punished for centrality, but also player 3 for being needed to reach player 4 .

Example 3.2 Take the star graph on four players with player 1 being the hub, as depicted in Figure 3.3.

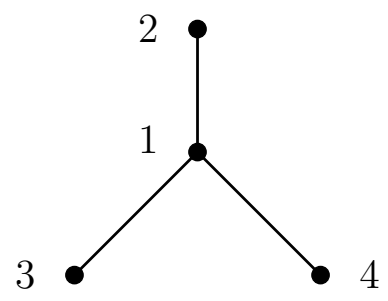

Figure 3.3

For this graph there are twelve consistent linear orderings, six with $\pi(1)=1$ and two with $\pi(j)=1$ for every $j \neq 1$. Any player $j, j \neq 1$, has the last position in four of the twelve linear orderings. Each player is the root of precisely one admissible spanning tree. There are sixteen admissible trees, one with player 1 as root and five with player $j, j \neq 1$, as root, see Figure 3.4 . 


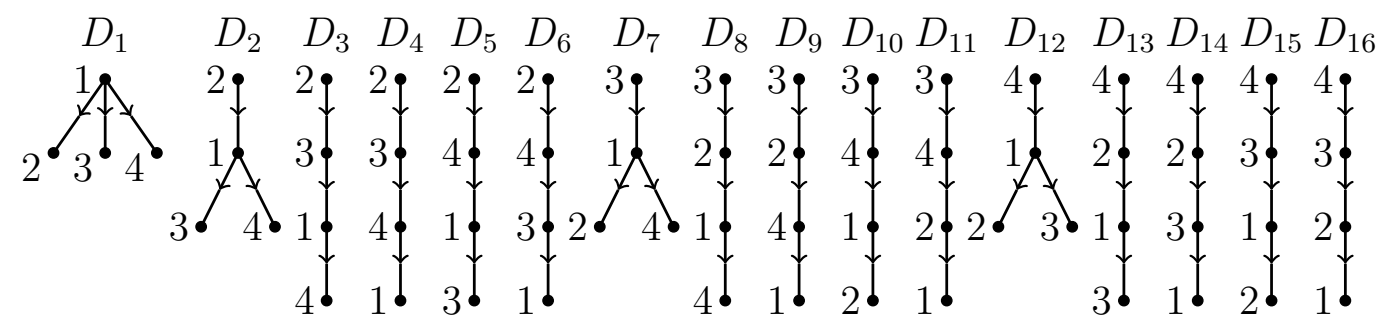

Figure 3.4

We consider again four different unanimity games, given by $u_{N}, u_{123}, u_{23}$, and $u_{12}$. The Myerson restricted games are given by $u_{N}^{\Gamma}=u_{N}, u_{23}^{\Gamma}=u_{123}^{\Gamma}=u_{123}$, and $u_{12}^{\Gamma}=u_{12}$. The payoff vectors of the four solutions are given in Table 3.2.

\begin{tabular}{|c|c|c|c|c|}
\hline$v$ & $\mu$ & $S h^{B}$ & $A T$ & $G C$ \\
\hline$u_{N}$ & $(1 / 4,1 / 4,1 / 4,1 / 4$ & $(0,1 / 3,1 / 3,1 / 3)$ & $(1 / 4,1 / 4,1 / 4,1 / 4)$ & $(1 / 16,5 / 16,5 / 16,5 / 16)$ \\
$u_{123}$ & $(1 / 3,1 / 3,1 / 3,0)$ & $(0,1 / 2,1 / 2,0)$ & $(1 / 2,1 / 4,1 / 4,0)$ & $(1 / 8,7 / 16,7 / 16,0)$ \\
$u_{23}$ & $(1 / 3,1 / 3,1 / 3,0)$ & $(0,1 / 2,1 / 2,0)$ & $(1 / 2,1 / 4,1 / 4,0)$ & $(1 / 8,7 / 16,7 / 16,0)$ \\
$u_{12}$ & $(1 / 2,1 / 2,0,0)$ & $(1 / 6,5 / 6,0,0)$ & $(3 / 4,1 / 4,0,0)$ & $(5 / 16,7 / 16,0,0)$ \\
\hline
\end{tabular}

Table 3.2

The Myerson value and the average tree solution reward for communication, but not for centrality. In particular both solutions give the same outcome for the two characteristic functions $u_{123}$ and $u_{23}$. Although the average tree solution gives in both case a higher payoff to player 1 than to players 2 and 3, this is not because player 1 is the hub but because player 1 also represents player 4. Again the solution of Bilbao does not reward for communication and punishes central player 1 . The gravity center rewards for communication, but punishes for centrality.

Example 3.3 Take $N=\{1,2,3,4\}$ and $\Gamma=\{\{1,2\},\{1,3\},\{1,4\},\{2,3\}\}$, as depicted in Figure 3.3.

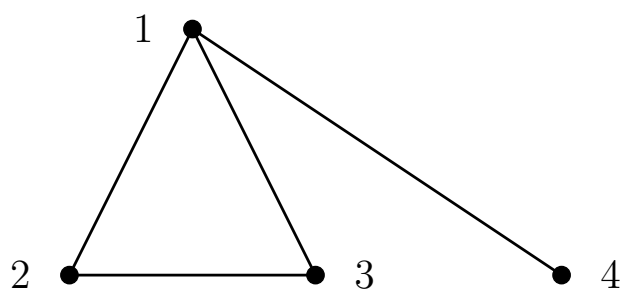

Figure 3.5 
For this graph there are fourteen consistent linear orderings, given by $\pi^{1}=(1,2,3,4)$, $\pi^{2}=(1,2,4,3), \pi^{3}=(1,3,2,4), \pi^{4}=(1,3,4,2), \pi^{5}=(1,4,2,3), \pi^{6}=(1,4,3,2), \pi^{7}=$ $(2,1,3,4), \pi^{8}=(2,1,4,3), \pi^{9}=(2,3,1,4), \pi^{10}=(3,1,2,4), \pi^{11}=(3,1,4,2), \pi^{12}=$ $(3,2,1,4), \pi^{13}=(4,1,2,3)$, and $\pi^{14}=(4,1,3,2)$.

There are eighteen admissible trees, with player 1 being the root of two trees, players 2 and 3 the root of five trees, and player 4 the root of six trees, as depicted in Figure 3.6. There are eight admissible spanning trees, in Figure 3.6 given by $D_{1}, D_{2}, D_{3}, D_{4}$, $D_{8}, D_{9}, D_{13}$, and $D_{14}$.

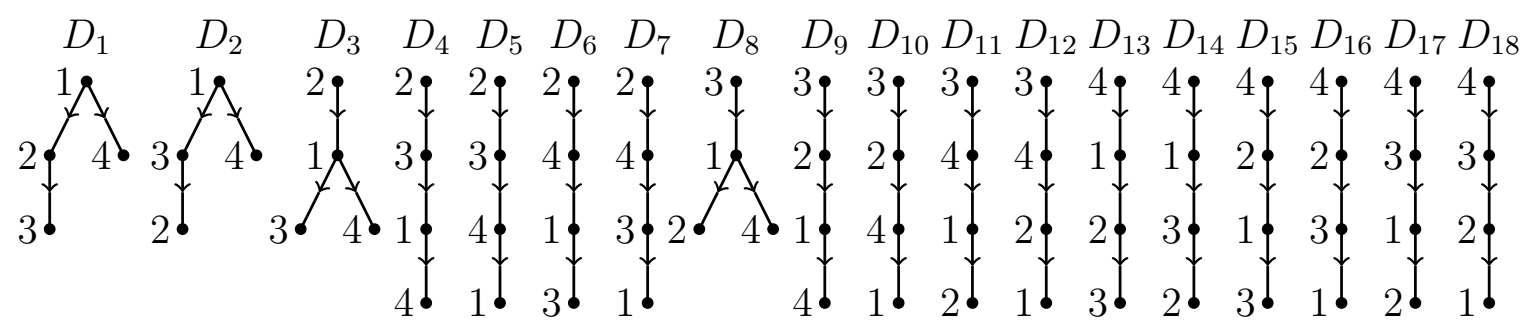

Figure 3.6

We consider the unanimity games $u_{N}, u_{123}, u_{124}$, and $u_{24}$. Their Myerson restricted games are given by $u_{N}^{\Gamma}=u_{N}, u_{123}^{\Gamma}=u_{123}$, and $u_{124}^{\Gamma}=u_{24}^{\Gamma}=u_{124}$. The payoff vectors of the four solutions are given in Table 3.3.

\begin{tabular}{|c|c|c|c|c|}
\hline$v$ & $\mu$ & $S h^{B}$ & $A T$ & $G C$ \\
\hline$u_{N}$ & $(1 / 4,1 / 4,1 / 4,1 / 4$ & $(0,2 / 7,2 / 7,3 / 7)$ & $(1 / 4,1 / 4,1 / 4,1 / 4)$ & $(2 / 18,5 / 18,5 / 18,6 / 18)$ \\
$u_{123}$ & $(1 / 3,1 / 3,1 / 3,0)$ & $(1 / 7,3 / 7,3 / 7,0)$ & $(1 / 2,1 / 4,1 / 4,0)$ & $(4 / 18,7 / 18,7 / 18,0)$ \\
$u_{124}$ & $(1 / 3,1 / 3,0,1 / 3)$ & $(0,3 / 7,0,4 / 7)$ & $(3 / 8,3 / 8,0,1 / 4)$ & $(3 / 18,7 / 18,0,8 / 18)$ \\
$u_{24}$ & $(1 / 3,1 / 3,0,1 / 3)$ & $(0,3 / 7,0,4 / 7)$ & $(3 / 8,3 / 8,0,1 / 4)$ & $(3 / 18,7 / 18,0,8 / 18)$ \\
\hline
\end{tabular}

\section{Table 3.3}

The Myerson value and the average tree solution reward for communication, but not for centrality. In this case the average tree solution gives for the characteristic functions $u_{124}$ and $u_{24}$ a higher payoff to players 1 and 2 than to player 4 , because these two players both represent player 3. Again the solution of Bilbao does not reward for communication and punishes for centrality and the gravity center rewards for communication, but punishes for centrality. In this case we could even say that the latter two solutions reward player 4 for not being connected with the players 2 and 3 .

To conclude this section we want to stress that the value of Bilbao takes the average over a subset of the marginal vectors of the original game. Since in every marginal vector a null player gets zero payoff, this value has the standard null player property. Since a null player in game $v$ might become a non-null player in the Myerson restricted game $v^{\Gamma}$, 
the Myerson value does not satisfy the null player property: A null player might get a non-zero payoff when he is needed to connect non-null players. Finally, in a hierarchical outcome the successor set of a player may not be connected. Taking this into account, formula (??) can be rewritten as

$$
h_{j}^{D}(v, \Gamma)=v\left(\widehat{S}_{D}(j)\right)-v^{L}\left(\cup_{h \in S_{D}(j)} \widehat{S}_{D}(h)\right), \quad j \in N
$$

This shows that also when applying the average tree solution or the gravity center a null player might get a non-zero payoff when he is needed to connect non-null players. We conclude that the value of Bilbao differs from the Shapley value by restricting the collection of orderings, but that it still satisfies the null-player property, whereas the other three values do not satisfy the null player property.

\section{Rewarding centrality}

In this section we propose two solutions on the class of connected graph games. The first one does still have the null player property and so it does not reward communication necessity of a player, but it rewards centrality in the graph. The second value rewards both communication necessity and centrality. The values are defined in Subsection 4.1, which also contains some results for specific cases. Explicit expressions of the values are given in Subsection 4.2.

\subsection{Definitions and results}

Given a connected graph $\Gamma \in \mathcal{G}_{N}^{\Gamma_{c}}$ on the set of players $N$, a linear ordering $\pi \in \Pi(N)$ is said to preserve connectivity when for every $i \in N$ it holds that the subgraph on the set $N \backslash P^{\pi}(i)$ is connected, i.e., every $i \in N$ is an extreme node of the subgraph $\left.\Gamma\right|_{N \backslash P^{\pi}(i)}$. Whereas in the standard Shapley interpretation a linear ordering is typically interpreted as the order in which the players form the grand coalition, we now consider a linear ordering as an order in which the players leave the grand coalition. The order preserves connectivity when subsequently extreme players leave and thus after a player leaves the remaining players are still connected. We call a linear ordering preserving connectivity a connectivity ordering and denote by $\Pi_{c}^{\Gamma}(N)$ the collection of these orderings with respect to $\Gamma$. Note that $\pi \in \Pi_{c}^{\Gamma}(N)$ if and only if $\pi$ is the reverse of a consistent linear ordering. On the class of connected graph games we now define the following solution.

Definition 4.1 On the class $\mathcal{G}_{N}^{\Gamma_{c}}$ of connected graph games the centrality rewarding Shapley value $S h^{c}$ is the solution given by

$$
S h^{c}(v, \Gamma)=\frac{1}{\left|\Pi_{c}^{\Gamma}(N)\right|} \sum_{\pi \in \Pi_{c}^{\Gamma}(N)} m^{\pi}(v), \quad(v, \Gamma) \in \mathcal{G}_{N}^{\Gamma_{c}} .
$$

$S h^{c}(v, \Gamma)$ is the average of the marginal vectors of game $v$ over all connectivity orderings with respect to $\Gamma$. It thus modifies the Shapley value in the sense that the average is not taken over all linear orderings, but only over those linear orderings that preserve connectivity of the graph. 
The second solution on the class of connected graph games is obtained by taking the average of the marginal vectors of the Myerson restricted game over all connectivity orderings.

Definition 4.2 On the class $\mathcal{G}_{N}^{\Gamma_{c}}$ of connected graph games the centrality rewarding Myerson value $\mu^{c}$ is the solution given by

$$
\mu^{c}(v, \Gamma)=\frac{1}{\left|\Pi_{c}^{\Gamma}(N)\right|} \sum_{\pi \in \Pi_{c}^{\Gamma}(N)} m^{\pi}\left(v^{\Gamma}\right), \quad(v, \Gamma) \in \mathcal{G}_{N}^{\Gamma_{c}} .
$$

Example 4.1 We apply both centrality rewarding values to the graph games considered in the Examples ??-??. For each of these graph games the outcome $S h^{c}(v, \Gamma)$ is given in Table 4.1 and the outcome $\mu^{c}(v, \Gamma)$ in Table 4.2 .

\begin{tabular}{|c|c||c|c||c|c|}
\hline$? ?$ & $S h^{c}$ & $? ?$ & $S h^{c}$ & $? ?$ & $S h^{c}$ \\
\hline$u_{N}$ & $(1 / 8,3 / 8,3 / 8,1 / 8)$ & $u_{N}$ & $(1 / 2,1 / 6,1 / 6,1 / 6)$ & $u_{N}$ & $(6 / 14,3 / 14,3 / 14,2 / 14)$ \\
$u_{124}$ & $(1 / 8,5 / 8,0,2 / 8)$ & $u_{123}$ & $(2 / 3,1 / 6,1 / 6,0)$ & $u_{123}$ & $(8 / 14,3 / 14,3 / 14,0)$ \\
$u_{123}$ & $(1 / 8,3 / 8,5 / 8,0)$ & $u_{23}$ & $(0,1 / 2,1 / 2,0)$ & $u_{124}$ & $(8 / 14,4 / 14,0,2 / 14)$ \\
$u_{13}$ & $(2 / 8,0,6 / 8,0)$ & $u_{12}$ & $(5 / 6,1 / 6,0,0)$ & $u_{24}$ & $(0,8 / 14,0,6 / 14)$ \\
\hline
\end{tabular}

Table 4.1

\begin{tabular}{|c|c||c|c||c|c|}
\hline$? ?$ & $\mu^{c}$ & $? ?$ & $\mu^{c}$ & $? ?$ & $\mu^{c}$ \\
\hline$u_{N}$ & $(1 / 8,3 / 8,3 / 8,1 / 8)$ & $u_{N}$ & $(1 / 2,1 / 6,1 / 6,1 / 6)$ & $u_{N}$ & $(6 / 14,3 / 14,3 / 14,2 / 14)$ \\
$u_{124}$ & $(1 / 8,3 / 8,3 / 8,1 / 8)$ & $u_{123}$ & $(2 / 3,1 / 6,1 / 6,0)$ & $u_{123}$ & $(8 / 14,3 / 14,3 / 14,0)$ \\
$u_{123}$ & $(1 / 8,3 / 8,1 / 2,0)$ & $u_{23}$ & $(2 / 3,1 / 6,1 / 6,0)$ & $u_{124}$ & $(8 / 14,4 / 14,0,2 / 14)$ \\
$u_{13}$ & $(1 / 8,3 / 4,1 / 2,0)$ & $u_{12}$ & $(5 / 6,1 / 6,0,0)$ & $u_{24}$ & $(8 / 14,4 / 14,0,2 / 14)$ \\
\hline
\end{tabular}

\section{Table 4.2}

The tables show that for unanimity graph games both values yield the same payoffs when the unanimity coalition is connected in the graph. For both values we also have that they satisfy the null player property when the unanimity coalition is connected. Since the centrality rewarding Myerson value takes marginal vectors with respect to the Myerson restricted game, it holds that $\mu^{c}(N, v, \Gamma)=\mu^{c}\left(N, v^{\Gamma}, \Gamma\right)$. So for the graph of Example ?? we have that $\mu^{c}\left(u_{124}, \Gamma\right)=\mu^{c}\left(u_{124}^{\Gamma}, \Gamma\right)=\mu^{c}\left(u_{N}, \Gamma\right)$ and for the graph of Example ?? we have that $\mu^{c}\left(u_{24}, \Gamma\right)=\mu^{c}\left(u_{24}^{\Gamma}, \Gamma\right)=\mu^{c}\left(u_{124}, \Gamma\right)$. This also implies that $\mu^{c}$ respects communication, for instance in the graph of Example ?? the payoff to player 1 when $v=u_{24}$ is the same as the payoff to player 1 when $v=u_{124}$. Although in the former game player 1 is a null player in the game, this player is needed for the communication between players 2 and 4 .

Table 4.2 shows that $\mu^{c}$ not only respects communication, but also rewards for centrality. In particular this can be seen when considering the payoff vectors in the three examples when $v=u_{N}$. For the graph of Example ?? the payoffs of the players 2 and 3 is higher than the payoffs of the extreme players 1 and 4, for the graph of Example ?? the payoffs of the central player 1 is higher than the payoff of the other players, and 
for the graph of Example ?? the payoff of the central player 1 is higher than the payoff of the players 2 and 3, which in turn are higher than the payoff of player 4. Similar observations can be made for the other characteristic functions. Table 4.1 shows that $S h^{c}$ also rewards centrality, but it does not reward for communication necessity. For instance, for the case that $v=u_{24}$ of Example ?? player 1 is a null player and gets zero payoff, although he connects players 2 and 4.

Taking the unanimity game on the grand coalition, the next two propositions show the centrality flavor of the centrality rewarding Shapley value for the special cases of a star graph and a linear graph. Since for a graph game $\left(u_{N}, \Gamma\right)$ the two centrality rewarding values yield the same outcome, the same results hold for the centrality rewarding Myerson value.

Proposition 4.1 For a star graph $\Gamma$ on $N$ with $i_{0} \in N$ being the hub it holds that

$$
S h_{i}^{c}\left(u_{N}, \Gamma\right)=\left\{\begin{array}{cl}
\frac{1}{2}, & i=i_{0}, \\
\frac{1}{2(n-1)}, & i \neq i_{0} .
\end{array}\right.
$$

Proof. For every connectivity ordering it holds that the first $n-2$ positions are occupied by satellites, and the hub is either on position $n-1$ or on position $n$. This implies that the hub is in the last position in half of the linear orderings in the set $\Pi_{c}^{\Gamma}(N)$. This gives payoff $\frac{1}{2}$ to the hub. Since all other players are symmetric, each one of them gets payoff $\frac{1}{2(n-1)}$.

In the graph game $\left(u_{N}, \Gamma\right)$ with $\Gamma$ being a star, all players get the same payoff $\frac{1}{n}$ when applying the Shapley value, the Myerson value, and the average tree solution, whereas the hub gets payoff 0 according to the solution of Bilbao. When we consider the gravity center, note that the number of admissible trees is increasing in the number of players, but there is only one admissible tree having the hub as its root. Since the payoffs are obtained by taking the average of the hierarchical outcomes over all admissible trees, the payoff to the hub tends to zero when the number of players goes to infinity.

Proposition 4.2 For the linear graph $\Gamma$ on $N$ given by $\Gamma=\{\{i, i+1\} \mid i=1, \ldots, n-1\}$ it holds that

$$
S h_{k}^{c}\left(u_{N}, \Gamma\right)=\frac{1}{2^{n-1}}\left(\begin{array}{c}
n-1 \\
k-1
\end{array}\right), \quad k \in N .
$$

Proof. For any $\pi \in \Pi(N)$, we have that $m_{k}^{\pi}\left(u_{N}\right)=1$ if $\pi(k)=n$, and $m_{k}^{\pi}\left(u_{N}\right)=0$ otherwise. A linear ordering with $\pi(k)=n$ is preserving connectivity if and only if $\pi(j)<\pi(j+1)$ for $j<k$ and $\pi(j)>\pi(j+1)$ for $j>k$. Hence, for each $k \in N$ the number of linear orderings $\pi \in \Pi_{c}^{\Gamma}(N)$ for which $\pi(k)=n$ is equal to $\left(\begin{array}{l}n-1 \\ k-1\end{array}\right)$. Since $\sum_{k=1}^{n}\left(\begin{array}{l}n-1 \\ k-1\end{array}\right)=2^{n-1}$, the result follows from equation (??).

The next corollary follows immediately and again shows that the centrality rewarding Shapley value rewards centrality. 
Corollary 4.1 For the linear graph on $N$ with $\Gamma=\{\{i, i+1\} \mid i=1, \ldots, n-1\}$ it holds that

$$
S h_{i}^{c}\left(u_{N}, \Gamma\right)=S h_{n-i}^{c}\left(u_{N}, \Gamma\right) \text { for all } i \in N
$$

and

$$
S h_{i}^{c}\left(u_{N}, \Gamma\right)<S h_{i+1}^{c}\left(u_{N}, \Gamma\right) \text { for all } i<\frac{n}{2} \text {. }
$$

Other values that reward centrality are the position value and the Harsanyi power solutions, we comment on these values in Section 6 .

\subsection{Explicit representations}

In this section we provide explicit expressions for the centrality rewarding values introduced in the previous section. Without loss of generality we restrict ourselves to the class of connected graphs. We first introduce some additional notation.

Let be given a connected graph $\Gamma \in \Gamma_{N}^{c}$. For $i \in N, \Pi_{i}(\Gamma) \subset \Pi_{c}^{\Gamma}(N)$ denotes the set of connectivity orderings such that $\pi(i)=n$, i.e., player $i$ is the last player in the ordering. Then the connectivity degree of player $i$ as introduced in Khmelnitskaya, van der Laan, and Talman (2016) is defined by $c_{i}(\Gamma)=\left|\Pi_{i}(\Gamma)\right|$, being the number of connectivity orderings with respect to $\Gamma$ in which player $i$ is the last index.

For $i \in N$, we denote $N_{i}^{\Gamma}=\{j \in N \mid\{i, j\} \in \Gamma\}$ as the set of neighbors of $i$ in $\Gamma$. Further, for a coalition $S \subseteq N, N_{i}^{\Gamma}(S)=N_{i}^{\Gamma} \cap S$ denotes the set of neighbors of $i$ inside $S$ and $N_{S}^{\Gamma}=\cup_{i \in S} N_{i}^{\Gamma}(N \backslash S)$ the set of nodes not in $S$ that are a neighbor of at least one node in $S$. For a proper coalition $S \subset N$, node $i \in S$ is a border node of $S$ if $N_{i}^{\Gamma}(N \backslash S) \neq \emptyset$, i.e., $i$ has at least one neighbor outside $S$. We denote the set of border nodes of $S$ by $\partial^{\Gamma} S$ and define $\operatorname{Int}^{\Gamma}(S)=S \backslash \partial^{\Gamma} S$ as the set of interior nodes of $S$ in $\Gamma$. For a border node $i \in \partial^{\Gamma} S$, a connected coalition $Q \subseteq N \backslash S$ is an $i$-neighboring coalition for $S$ if $Q \cap N_{i}^{\Gamma} \neq \emptyset$, i.e., $Q$ contains at least one neighbor of $i$. For $i \in \partial^{\Gamma} S$, we denote by $((N \backslash S) / \Gamma)^{i}$ the collection of $i$-neighboring components of the complement $N \backslash S$ and for an $i$-neighboring component $C \in((N \backslash S) / \Gamma)^{i}$ we denote by $R_{i}^{\Gamma}(C)$ the set of $i$-neighboring connected coalitions that are a subset of $C$. For $C \in(N \backslash S) / \Gamma$, the extended subgraph of $\Gamma$ on $C$ with respect to $S$ is the graph $\Gamma_{C}^{S}$ on $C$ given by

$$
\Gamma_{C}^{S}=\left.\Gamma\right|_{C} \cup\left\{\{i, j\} \mid i, j \in C \cap N_{S}^{\Gamma}, i \neq j\right\} .
$$

The extended subgraph $\Gamma_{C}^{S}$ is the union of the subgraph $\left.\Gamma\right|_{C}$ and the set of links between any two neighbors of $S$ that belong to $C$. The idea of this definition of extended subgraph is based on the fact that all neighbors of $S$ in $C$ are connected to each other outside $C$ via nodes of $S$, and so, when we consider the subgraph on $C$, these neighbors appear as directly connected in $C$. Remark that $\Gamma_{C}^{S}=\left.\Gamma\right|_{C}$ when $C$ contains only one neighbor of $S$.

We first consider the centrality rewarding Shapley value. Since it is the average over a collection of marginal vectors, it follows immediately that it is a linear value. Hence, for any $(v, \Gamma) \in \mathcal{G}_{N}^{\Gamma_{c}}$ we have that

$$
S h^{c}(v, \Gamma)=\sum_{S \subseteq N, S \neq \emptyset} \lambda_{S}(v) S h^{c}\left(u_{S}, \Gamma\right) .
$$


Theorem 4.1 For any $\left(u_{S}, \Gamma\right) \in \mathcal{G}_{N}^{\Gamma_{c}}, \emptyset \neq S \subseteq N$, and every $i \in S$

$$
\begin{aligned}
& S h_{i}^{c}\left(u_{S}, \Gamma\right)=\frac{1}{\sum_{j \in N} c_{j}(\Gamma)}\left[c_{i}(\Gamma)+\right. \\
& \left.\quad \sum_{C \in((N \backslash S) / \Gamma)^{i}} \sum_{Q \in R_{i}^{\Gamma}(C)}\left[\sum_{j \in Q} c_{j}\left(\left.\Gamma\right|_{Q}\right)\right]\left[\left(\begin{array}{c}
|N \backslash Q|-1 \\
|K|, K \in\left(N \backslash Q_{+i}\right) / \Gamma
\end{array}\right) \prod_{K \in\left(N \backslash Q_{+i}\right) / \Gamma} \sum_{h \in N_{Q_{+i}}^{\Gamma} \cap K} c_{h}\left(\Gamma_{K}^{Q_{+i}}\right)\right]\right],
\end{aligned}
$$

and $S h_{i}^{c}\left(u_{S}, \Gamma\right)=0$ for every $i \in N \backslash S$.

Proof. For any linear ordering $\pi \in \Pi(N)$, the marginal contribution $m_{i}^{\pi}\left(u_{S}\right)=u_{S}\left(\bar{P}^{\pi}(i)\right)-$ $u_{S}\left(P^{\pi}(i)\right)=1$ if and only if $i \in S$ and $S \subseteq \bar{P}^{\pi}(i)$, otherwise $m_{i}^{\pi}\left(u_{S}\right)=0$. From this it follows immediately that $S h_{i}^{c}\left(u_{S}, \Gamma\right)=0$ when $i \in N \backslash S$. Moreover, for $i \in S$ and connectivity ordering $\pi \in \Pi_{c}^{\Gamma}(N), m_{i}^{\pi}\left(u_{S}\right)=1$ holds only if either $\pi(i)=n$, or $i \in \partial^{\Gamma} S$, $((N \backslash S) / \Gamma)_{\pi^{-1}(n)} \in((N \backslash S) / \Gamma)^{i}$, and $N \backslash \bar{P}^{\pi}(i) \in R_{i}^{\Gamma}\left(((N \backslash S) / \Gamma)_{\pi^{-1}(n)}\right)$. Besides, the subordering of $\pi$ on $N \backslash \bar{P}^{\pi}(i)$ is a connectivity ordering on the subgraph $\left.\Gamma\right|_{N \backslash \bar{P}^{\pi}(i)}$. In a connectivity ordering $\pi \in \Pi_{c}^{\Gamma}(N)$, player $i$ is preceded by one of the neighbors of the set $N \backslash P^{\pi}(i)$, not belonging to this set, and each of these neighbors is connected to $i$ via nodes in $N \backslash P^{\pi}(i)$. From the connectedness of $\Gamma$ it follows that every component $\left.C \in P^{\pi}(i)\right) / \Gamma$ contains at least one neighbor of $N \backslash P^{\pi}(i)$ and when $\Gamma$ is not cycle-free, $C$ may contain more than one neighbor of this set. In the latter case each pair of neighbors of $N \backslash P^{\pi}(i)$ in $C$ is connected both in the component $C$ and outside $C$ via the nodes of $N \backslash P^{\pi}(i) \subseteq N \backslash C$. For $\pi \in \Pi_{c}^{\Gamma}(N)$ and $\left.C \in P^{\pi}(i)\right) / \Gamma$, the linear subordering of $\pi$ on $C$ starts with a neighbor $h \in C \cap N_{N \backslash P^{\pi}(i)}^{\Gamma}$. If $\left|C \cap N_{N \backslash P^{\pi}(i)}^{\Gamma}\right|=1$ the subordering of $\pi$ on $C$ satisfies connectivity on the subgraph $\left.\Gamma\right|_{C}$. When $C \cap N_{N \backslash P^{\pi}(i)}^{\Gamma}$ contains more than one neighbor of $N \backslash P^{\pi}(i)$, then in the subordering of $\pi$ on $C$ these neighbors being connected to each other via their predecessors in the set $N \backslash P^{\pi}(i)$ may follow each other in any order, which is equivalent that the subordering of $\pi$ on $C$ satisfies connectivity on the extended subgraph $\Gamma_{C}^{N \backslash P^{\pi}(i)}$. Recall that if $\left|C \cap N_{N \backslash P^{\pi}(i)}^{\Gamma}\right|=1$, then $\Gamma_{C}^{N \backslash P^{\pi}(i)}=\left.\Gamma\right|_{C}$, and therefore both cases $\left|C \cap N_{N \backslash P^{\pi}(i)}^{\Gamma}\right|=1$ and $\left|C \cap N_{N \backslash P^{\pi}(i)}^{\Gamma}\right|>1$ may be considered in the unique framework of the extended subgraph. For any $\left.C \in P^{\pi}(i)\right) / \Gamma$ and $h \in C \cap N_{N \backslash P^{\pi}(i)}^{\Gamma}$ there are exactly $c_{h}\left(\Gamma_{C}^{N \backslash P^{\pi}(i)}\right)$ connectivity orderings on $C$ that start with $h$, which gives in total $\sum_{h \in C \cap N_{N \backslash P^{\pi}(i)}^{\Gamma}} c_{h}\left(\Gamma_{C}^{N \backslash P^{\pi}(i)}\right)$ connectivity orderings on $C$. Since the components $\left.C \in P^{\pi}(i)\right) / \Gamma$ are not connected to each other, the connectivity of an ordering does not depend on the order of nodes in different components. Wherefrom it follows that the total number of connectivity orderings $\pi \in \Pi_{c}^{\Gamma}(N)$ for which $m_{i}^{\pi}\left(u_{S}\right)=1, i \in S$, is equal to

$$
c_{i}(\Gamma)+\sum_{C \in((N \backslash S) / \Gamma)^{i}} \sum_{Q \in R_{i}^{\Gamma}(C)}\left[\sum_{j \in Q} c_{j}\left(\left.\Gamma\right|_{Q}\right)\right]\left[\left(\begin{array}{c}
|N \backslash Q|-1 \\
|K|, K \in\left(N \backslash Q_{+i}\right) / \Gamma
\end{array}\right) \prod_{K \in\left(N \backslash Q_{+i}\right) / \Gamma} \sum_{h \in N_{Q_{+i}}^{\Gamma} \cap K} c_{h}\left(\Gamma_{K}^{Q_{+i}}\right)\right] .
$$

Then formula (??) follows immediately from the fact that $\left|\Pi_{c}^{\Gamma}(N)\right|=\sum_{i \in N} c_{i}(\Gamma)$. 
Remark 4.1 Note that in formula (??) the collection $((N \backslash S) / \Gamma)^{i}$ is empty when $i \in$ $\operatorname{Int}^{\Gamma}(S)$. Hence, for such a player $i$ the formula simply reduces to $S h_{i}^{c}\left(u_{S}, \Gamma\right)=\frac{c_{i}(\Gamma)}{\sum_{j \in N} c_{j}(\Gamma)}$. In particular, for the unanimity graph game $\left(u_{N}, \Gamma\right)$ on the grand coalition, equation (??) reduces to

$$
S h_{i}^{c}\left(u_{N}, \Gamma\right)=\frac{c_{i}(\Gamma)}{\sum_{j \in N} c_{j}(\Gamma)}, i \in N .
$$

In this case the payoff to a player is proportional to its connectivity degree.

Remark 4.2 For a connected cycle-free graph game $\left(u_{S}, \Gamma\right) \in \mathcal{G}_{N}^{\Gamma_{c f}}$ formula (??) reduces to

$$
\begin{aligned}
& S h_{i}^{c}\left(u_{S}, \Gamma\right)=\frac{1}{\sum_{j \in N} c_{j}(\Gamma)}\left[c_{i}(\Gamma)+\right. \\
& \left.\quad \sum_{C \in((N \backslash S) / \Gamma)^{i}} \sum_{Q \in R_{i}^{\Gamma}(C)}\left[\sum_{j \in Q} c_{j}\left(\left.\Gamma\right|_{Q}\right)\right]\left[\left(\begin{array}{c}
|N \backslash Q|-1 \\
|K|, K \in\left(N \backslash Q_{+i}\right) / \Gamma
\end{array}\right) \prod_{h \in N_{Q_{+i}}^{\Gamma}} c_{h}\left(\left.\Gamma\right|_{\left(\left(N \backslash Q_{+i}\right) / \Gamma\right)_{h}}\right)\right]\right] .
\end{aligned}
$$

Further, if $\left(u_{S}, \Gamma\right) \in \mathcal{G}_{N}^{\Gamma_{c f}}$, then for every connected coalition $S$ we have that each component $C$ of the subgraph $\left.\Gamma\right|_{N \backslash S}$ has precisely one neighbor in $S$. In that case we call any component in $\left.\Gamma\right|_{N \backslash S}$ a satellite of $S$. Then in the payoff vector for the game $\left(u_{S}, \Gamma\right)$ all the payoffs that the players in a component $C$ in the subgraph $\left.\Gamma\right|_{N \backslash S}$ receive in the game $\left(u_{N}, \Gamma\right)$ are transferred to the unique neighbor of $C$ in $S$. So, when $\Gamma$ is connected and cycle-free and $S$ is connected, formula (??) reduces to

$$
S h_{i}^{c}\left(u_{S}, \Gamma\right)= \begin{cases}S h_{i}^{c}\left(u_{N}, \Gamma\right) & \text { if } i \in \operatorname{Int}^{\Gamma}(S), \\ \frac{1}{\sum_{j \in N} c_{j}(\Gamma)}\left[c_{i}(\Gamma)+\sum_{C \in((N \backslash S) / \Gamma)^{i}} \sum_{j \in C} c_{j}(\Gamma)\right] & \text { if } i \in \partial^{\Gamma} S .\end{cases}
$$

Example 4.2 We consider the graph game $\left(u_{S}, \Gamma\right)$ with $\Gamma$ with linear graph $\Gamma=\{\{i, i+$ $1\} \mid i=1, \ldots, n-1\}$. Every connected coalition $Q \subseteq N$ is of the form $Q=\{i, \ldots, i+l\}$ for some $i \geq 1$ and $0 \leq l \leq n-i$. Therefore, each component $C \in S \backslash \Gamma$ has at most two border nodes. For $C=\{i, \ldots, i+l\} \in S \backslash \Gamma$ we call $i$ the left border node of $C$ if $i \neq 1$ and $i+l$ the right border node of $C$ if $i+l \neq n$. We denote by $\partial_{r}^{\Gamma} S\left(\partial_{l}^{\Gamma} S\right)$ the collection of right (left) border nodes of the components of $S \backslash \Gamma$. Note that node $i$ belongs to both when $\{i\}$ is a singleton component in $S \backslash \Gamma$ (and $i \neq 1, n$ ). Every node $i \in \partial_{r}^{\Gamma} S$ has a unique $i$-neighboring component in $(N \backslash S) / \Gamma$ to its right, similarly every $i \in \partial_{l}^{\Gamma} S$ has a unique $i$-neighboring component in $(N \backslash S) / \Gamma$ to its left. Consider a right border node $i$ and let $C=\{i+1, \ldots, i+l\}$ be the corresponding $i$-neighboring component in $N \backslash S$. Then every $i$-neighboring coalition $Q$ in $C$ has the form $\{i+1, \ldots, i+h\}$ for some $h, 1 \leq h \leq l$, i.e., the subgraph $\Gamma_{Q}$ is a linear graph with $h$ nodes. As shown in Khmelnitskaya, van der Laan, and Talman (2016), the connectivity degree of a node 
$j$ of $Q$ in the subgraph $\left.\Gamma\right|_{Q}$ is given by $c_{j}\left(\left.\Gamma\right|_{Q}\right)=\left(\begin{array}{c}h-1 \\ j-1\end{array}\right)$, i.e., $c_{j}\left(\left.\Gamma\right|_{Q}\right)$ is the binomial coefficient $C_{h-1}^{j-1}=\left(\begin{array}{c}h-1 \\ j-1\end{array}\right)$. Hence $\sum_{j \in Q} c_{j}\left(\left.\Gamma\right|_{Q}\right)=\sum_{j=1}^{h-1} C_{h-1}^{j-1}=2^{h-1}$. Moreover, for every $h \in N_{Q_{+i}}^{\Gamma}$, we have that $c_{h}\left(\left.\Gamma\right|_{\left(\left(N \backslash Q_{+i}\right) / \Gamma\right)_{h}}\right)=1$. Hence, equation (??) reduces to

$$
S h_{i}^{c}\left(u_{S}, \Gamma\right)=\frac{1}{\sum_{j \in N} c_{j}(\Gamma)}\left[c_{i}(\Gamma)+\sum_{h=1}^{l} 2^{h-1} C_{n-h-1}^{i-1}\right] .
$$

The case of $i$ being a left border node is very similar. Since $c_{i}(\Gamma)=C_{n-1}^{i-1}$ for all $i \in N$, we obtain

$$
S h_{i}^{c}\left(u_{S}, \Gamma\right)= \begin{cases}0, & i \notin S, \\ \frac{C_{n-1}^{i-1},}{2^{n-1}} & i \in \operatorname{Int}^{\Gamma}(S), \\ \frac{C_{n-1}^{i-1}+\sum_{h=1}^{r_{i}} 2^{h-1} C_{n-h-1}^{i-1}}{2^{n-1}}, & i \in \partial_{r}^{\Gamma} S,\left|(S / \Gamma)_{i}\right| \geq 2, \\ \frac{C_{n-1}^{i-1}+\sum_{h=1}^{l_{i}} 2^{h-1} C_{n-h-1}^{i-h}}{2^{n-1}}, & \text { or }\{i\} \in S / \Gamma, i=1, \\ \frac{C_{n-1}^{i-1}+\sum_{h=1}^{r_{i}} 2^{h-1} C_{n-h-1}^{i-1}+\sum_{h=1}^{l_{i}} 2^{h-1} C_{n-h-1}^{i-h}}{2^{n-1}}, & \text { or }\{i\} \in S / \Gamma, i=n,\left|(S / \Gamma)_{i}\right| \geq 2, \\ \frac{}{2} & \end{cases}
$$

where $r_{i}=\left|((N \backslash S) / \Gamma)_{i+1}\right|$ and $l_{i}=\left|((N \backslash S) / \Gamma)_{i-1}\right|$ for appropriate $i \in N$.

Next we consider the centrality rewarding Myerson value. We now have that for every $(v, \Gamma) \in \mathcal{G}_{N}^{\Gamma_{c}}$ it holds that

$$
\mu^{c}(v, \Gamma)=\sum_{S \subseteq N, S \neq \emptyset} \lambda_{v^{\Gamma}}(S) \mu^{c}\left(u_{S}, \Gamma\right) .
$$

Since $\lambda_{v}(S)=0$ when $S$ is not connected, we only have to consider the unanimity graph games $\left(u_{S}, \Gamma\right)$ for connected coalitions $S$. For these coalitions we have that $\mu^{c}\left(u_{S}, \Gamma\right)=$ $S h^{c}\left(u_{S}, \Gamma\right)$ and so the centrality rewarding Myerson payoffs for the connected coalitions $S$ also follow from Theorem ??. Note that again Remark ?? and the second statement in Remark ?? hold.

\section{Axiomatization of the centrality rewarding values on the class of connected cycle-free graph games}

In this section we provide an axiomatic characterization of both centrality rewarding values on the class of connected cycle-free graph games. In the first subsection we consider the centrality rewarding Myerson value, in the second one the centrality rewarding Shapley value. 


\subsection{The centrality rewarding Myerson value}

We characterize the centrality rewarding Myerson value on the subclass $\mathcal{G}_{N}^{\Gamma_{c f}}$ of connected, cycle-free graph games by means of six independent axioms, some axioms being standard within the theory of cooperative games, some of them are similar to axioms known from the literature of the average tree solution, and some of them are modifications from axioms for permission values on the class of directed tree games. As for the average tree solution, characterization of the centrality rewarding Myerson value on the class of all connected graph games remains an open problem. Let $f$ be a value on $\mathcal{G}_{N}^{\Gamma_{c f}}$.

The standard axiom of efficiency states that the total sum of payoffs is equal to the worth of the grand coalition.

\section{Efficiency}

For any $(v, \Gamma) \in \mathcal{G}_{N}^{\Gamma_{c f}}$ it holds that $\sum_{i \in N} f_{i}(v, \Gamma)=v(N)$.

Linearity is a straightforward generalization of the linearity axiom for TU games.

\section{Linearity}

For any $(v, \Gamma),(w, \Gamma) \in \mathcal{G}_{N}^{\Gamma_{c f}}$ and real numbers $\alpha$ and $\beta$ it holds that $f(\alpha v+\beta w, \Gamma)=$ $\alpha f(v, \Gamma)+\beta f(w, \Gamma)$.

As usual a player $i \in N$ is called a null player in a TU game $v \in \mathcal{G}_{N}$ if for all $T \subset N \backslash\{i\}$ it holds that $v(T \cup\{i\})-v(T)=0$. In a connected cycle-free graph game $(v, \Gamma)$ player $i \in N$ is said to be a dummy intermediate if for every two non-null players $h$ and $k$, different from $i$, player $i$ is not on the path between $h$ and $k$. So, it means that a dummy intermediate is never needed to connect two other non-null players, and therefore he does not play a role in the communication between any pair of non-null players. We say that player $i$ is inessential for $(v, \Gamma)$ when $i$ is both a null player in $v$ and a dummy intermediate in $(v, \Gamma)$. The next axiom states that inessential players earn zero payoff. The axiom modifies the inessential player axiom as used in van den Brink, Herings, van der Laan, and Talman (2015) on the class of permission tree games to the class of connected cycle-free graph games. Note that the axiom weakens the usual null player property, which states that a null player earns zero payoff.

\section{Inessential player property}

For any $(v, \Gamma) \in \mathcal{G}_{N}^{\Gamma_{c f}}$ it holds that for every inessential player $i \in N, f_{i}(v, \Gamma)=0$.

Many values are axiomatized by some type of symmetry axiom. A weak version of such an axiom is that all players get the same payoff when the game is a multiple of the unanimity game on the grand coalition. However, although in this case the players are symmetric in the game, typically players have asymmetric positions in the communication graph. Thus it is not straightforward to use symmetry axioms in case of graph games, because such axioms typically ignore the communication structure. In the next axiom the distribution of the payoffs when $v=u_{N}$ reflects the positions of the players in the communication graph. Given a link $\{k, h\} \in \Gamma$, for $j=k, h$, let $N_{j}(\Gamma \backslash\{k, h\})$ be the set of players in the component of the graph $(N, \Gamma \backslash\{k, h\})$ that contains $j, j=k, h$. 
Note that $N_{h}(\Gamma \backslash\{k, h\}) \cup N_{k}(\Gamma \backslash\{k, h\})=N$. Then the next axiom states that the ratio of the payoffs between two neighbors $k$ and $h$ in a graph game $\left(u_{N}, \Gamma\right)$ is equal to the ratio of the number of players in their component when the link $\{k, h\}$ is removed from $\Gamma$.

\section{Communication fairness}

For any $\left(u_{N}, \Gamma\right) \in \mathcal{G}_{N}^{\Gamma_{c f}}$ and $\{k, h\} \in \Gamma$ it holds that

$$
\frac{f_{k}\left(u_{N}, \Gamma\right)}{\left|N_{k}(\Gamma \backslash\{k, h\})\right|}=\frac{f_{h}\left(u_{N}, \Gamma\right)}{\left|N_{h}(\Gamma \backslash\{k, h\})\right|} .
$$

The next axiom reflects the communication necessity of a player when he is on the path between two non-null players. It states that when $i \notin T$ is a neighbor of $j \in T$ on the path to another $k \in T$, then $i$ is necessary for $j$ in the sense that the payoff distribution in $\left(u_{T}, \Gamma\right)$ is equal to the payoff distribution in $\left(u_{T \cup\{i\}}, \Gamma\right)$. The axiom modifies the predecessor necessity axiom as used in van den Brink et al. (2015) on the class of permission tree games to the class of connected cycle-free graph games. This predecessor necessity axiom for permission tree games requires that the outcome does not change when a unanimity game $u_{T}$ is replaced by the unanimity game $u_{T \cup\{i\}}$ for any player $i$ being a predecessor in the tree of a player in $T$. Here, for $T \subset N$, let $H(T)$ denote the set of players that are on a path between two members of $T$. We say that $H(T)$ is the connected hull of $T$. Then the axiom requires that the outcome does not change when $u_{T}$ is replaced by $u_{T \cup\{i\}}$ for any $i \in H(T) \backslash T$ that is a neighbor of a player in $T$.

\section{Inside neighbor necessity}

For any $T \subset N,\left(u_{T}, \Gamma\right) \in \mathcal{G}_{N}^{\Gamma_{c f}}$, and $j \in T$ and $i \in H(T) \backslash T$ such that $\{i, j\} \in \Gamma$, it holds that $f\left(u_{T}, \Gamma\right)=f\left(u_{T \cup\{i\}}, \Gamma\right)$.

The axioms of inessential player property and inside neighbors necessity are together weaker than the axiom of restricted null player property used in Suzuki (2015, Chapter 2) to characterize the Myerson value. ${ }^{1}$ So, the restricted null player property implies the inessential player property and inside neighbor necessity, but not the other way around. For instance, the restricted null player property implies that every player gets zero payoff when $v(S)=0$ for every connected $S$, but this does not follow from the inessential player property and inside neighbor necessity together.

For TU games, Haller (1994) considers collusion neutrality properties, one of them stating that when two players are going to act together in the sense that the marginal contribution of a player to a coalition that does not contain the other player is made equal to zero, then the sum of the payoffs of the two players does not change. A different, but equivalent collusion neutrality property was introduced by Malawski (2002). In van den Brink (2012) it is shown that on the class of TU games there is no solution that satisfies efficiency, the null player property, and collusion neutrality, but that on the class of communication graph games all hierarchical outcomes and their convex combinations satisfy these three properties when only cooperation is allowed among neighbors. For a

\footnotetext{
${ }^{1}$ In Mishra and Talman (2010) the restricted null player property is called dummy and in van den Brink (2009) it is called the superfluous player property. In both studies the property is used to characterize the average tree solution on the class of connected cycle-free graph games.
} 
game $v \in \mathcal{G}$ and two players $i, j \in N$, the game in which players $i$ and $j$ act together is defined as the game $v^{i j} \in \mathcal{G}$ given by

$$
\begin{aligned}
v^{i j}(T) & =v(T \backslash\{i, j\}) & & \text { if }\{i, j\} \not \subset T \\
& =v(T) & & \text { otherwise. }
\end{aligned}
$$

The collusion property then states that $f_{i}(v)+f_{j}(v)=f_{i}\left(v^{i j}\right)+f_{j}\left(v^{i j}\right)$.

When applied to a unanimity game $u_{T}, T \in 2^{N}$, the colluded game $u_{T}^{i j}$ becomes

$$
\begin{aligned}
u_{T}^{i j} & =u_{T} & & \text { if }\{i, j\} \subseteq N \backslash T, \\
& =u_{T \cup\{i, j\}} & & \text { otherwise. }
\end{aligned}
$$

Therefore, for a unanimity game $u_{T}, j \in T$, and $i \notin T$, the collusion neutrality property reduces to $f_{i}\left(u_{T}\right)+f_{j}\left(u_{T}\right)=f_{i}\left(u_{T \cup\{i\}}\right)+f_{j}\left(u_{T \cup\{i\}}\right)$. For a graph game $\left(u_{T}, \Gamma\right)$ we only consider connected sets $T$ and players $j \in T$ and $i \notin T$ such that $\{i, j\} \in \Gamma$. On the other hand, the next axiom is stronger than the collusion property in the sense that when two neighbors, one in $T$ and the other not, collude, the payoffs to all other players in $T$ do not change. Note that for a connected $T$ every player $h \notin T \cup\{i\}$ is inessential in both $\left(u_{T}, \Gamma\right)$ and $\left(u_{T \cup\{i\}}, \Gamma\right)$. So, together with the inessential axiom, the next axiom implies that the joint payoff of the players $i$ and $j$ does not change. In fact, the next axiom coinides with the independence in unanimity games axiom used in Mishra and Talman (2010) to characterize the average tree solution.

\section{Independence of joining outside neighbors}

For any connected $T \subset N,\left(u_{T}, \Gamma\right) \in \mathcal{G}_{N}^{\Gamma_{c f}}$, and $j \in T$ and $i \in N \backslash T$ such that $\{i, j\} \in \Gamma$, it holds that $f_{k}\left(u_{T}, \Gamma\right)=f_{k}\left(u_{T \cup\{i\}}, \Gamma\right)$ for all $k \in T \backslash\{j\}$.

The next proposition states that the six axioms determine a unique solution.

Proposition 5.1 On the class of connected cycle-free graph games there is a unique solution that satisfies efficiency, linearity, the inessential player property, communication fairness, inside neighbor necessity, and independence of joining outside neighbors.

Proof. The proof goes in four steps. ${ }^{2}$

(i) For every connected cycle-free graph $\Gamma$, the payoff vector $f\left(u_{N}, \Gamma\right)$ is unique determined by efficiency and communication fairness.

(ii) For connected $T \subset N$, consider the unanimity graph game $\left(u_{T}, \Gamma\right)$. Then there exists a sequence of connected sets $T^{0}, \ldots, T^{r}$ with $r=|N \backslash T|$, such that $T^{0}=N, T^{r}=T$ and $T^{k}=T^{k-1} \backslash\left\{t_{k}\right\}$ for some $t_{k} \in T^{k-1} \backslash T, k=1, \ldots, r$. Since every $T^{k}$ is connected, any $i$ not in $T^{k}$ is inessential in the game $\left(u_{T^{k}}, \Gamma\right)$ and so $f_{i}\left(u_{T^{k}}, \Gamma\right)=0$. Then, starting from $f\left(u_{N}, \Gamma\right)$, the payoffs of the players $i \in T$ follow uniquely by repeated application for $k=1, \ldots, r$ of the independence of joining outside members.

(iii) For $T \subset N$ not connected, it follows from repeated application of inside neighbor necessity that $f\left(u_{T}, \Gamma\right)=f\left(u_{H(T)}, \Gamma\right)$. Since $H(T)$ is connected, this determines $f\left(u_{T}, \Gamma\right)$.

\footnotetext{
${ }^{2}$ We only give a scetch of the proof. Similar proofs for solutions on the class of directed tree games are given in more detail in e.g. van den Brink et al. (2015) and van den Brink et al. (2016).
} 
(iv) By the steps (i)-(iii) the payoffs are uniquely determined for every unanimity graph game $\left(u_{T}, \Gamma\right), \emptyset \neq T \subseteq N$. Then by linearity $f(v, \Gamma)$ is uniquely determined for any $(v, \Gamma) \in \mathcal{G}_{N}^{\Gamma_{c f}}$.

Next we show that the centrality rewarding Myerson value $\mu^{C}$ satisfies the six axioms on the class of connected cycle-free graph games. Let $(v, \Gamma) \in \mathcal{G}_{N}^{\Gamma_{c f}}$. Since $\Gamma$ is connected and cycle-free it follows that for any $\pi \in \Pi_{i}(\Gamma)$ and $h \in N$, the set $P^{\pi}(h)$ contains all players that are the subordinates of $h$ in the unique admissible spanning tree having player $i$ as root. Therefore we have for any $\pi \in \Pi_{i}(\Gamma)$ that

$$
m^{\pi}(v, \Gamma)=h^{D_{i}}(v, \Gamma)
$$

where $D_{i}$ denotes the admissible spanning tree on $N$ with root node $i$. It now follows that

$$
\mu^{c}(N, v, \Gamma)=\sum_{i \in N} \frac{c_{i}(\Gamma)}{\left|\Pi_{c}^{\Gamma}(N)\right|} h^{D_{i}}(N, v, \Gamma),
$$

i.e., on the class of connected cycle-free graph games the centrality rewarding Myerson value assigns the weighted average of the hierarchical outcomes with the weight of hierarchical outcome with respect to tree $D_{i}$ equal to the fraction of connectivity orderings having player $i$ as last player. The following lemma has been proven in Khmelnitskaya, van der Laan, and Talman (2016).

Lemma 5.1 Let $\Gamma$ be a connected, cycle-free graph. Then for every $k, h$ with $\{k, h\} \in \Gamma$ it holds that

$$
\frac{c_{k}(\Gamma)}{c_{h}(\Gamma)}=\frac{\left|N_{k}(\Gamma \backslash\{k, h\})\right|}{\left|N_{h}(\Gamma \backslash\{k, h\})\right|} .
$$

We are now ready to prove the next proposition.

Proposition 5.2 On the class of connected cycle-free graph games the centrality rewarding Myerson value satisfies efficiency, linearity, the inessential player property, communication fairness, inside neighbor necessity, and independence of joining outside neighbors.

\section{Proof.}

1. By definition every hierarchical outcome is efficient. Since $\sum_{h \in N} c_{h}(\Gamma)=\left|\Pi_{c}^{\Gamma}(N)\right|$, it follows that $\mu^{c}$ is efficient.

2. Consider two games $v, w \in \mathcal{G}$, real numbers $\alpha, \beta$, and define $z=\alpha v+\beta w$. Since for every $D \in T^{s}(\Gamma)$ the hierarchical outcome $h^{D}(v, \Gamma)$ is linear in its first argument, it follows that $h^{D}(z, \Gamma)=h^{D}(\alpha v+\beta w, \Gamma)=\alpha h^{D}(v, \Gamma)+\beta h^{D}(w, \Gamma)$. Since the centrality rewarding Myerson value is the weighted sum of all $n$ hierarchical outcomes, it follows that $\mu^{c}$ is linear.

3. Recall that any characteristic function $v$ can be written as $v=\sum_{T \subseteq N, T \neq \emptyset} \lambda_{T}(v) u_{T}$ with $\lambda_{T}(v)$ the Harsanyi dividend of coalition $T$ in TU game $v$. Then $k$ is inessential in $(v, \Gamma)$ if and only if $k$ is inessential in every $\left(u_{T}, \Gamma\right)$ with $\lambda_{T}(V) \neq 0$. Consider such a 
game $\left(u_{T}, \Gamma\right)$ and any hierarchical outcome $h^{D}\left(u_{T}, \Gamma\right)$. Then either every $j \in T$ is a successor of $k$ in $D$ or every $j \in T$ is a predecessor of $k$ in $D$. In both cases $h_{k}^{D}\left(u_{T}, \Gamma\right)=0$. By the linearity it follows that $\mu_{k}^{c}(v, \Gamma)=0$.

4. For the unanimity graph game $\left(u_{N}, \Gamma\right)$ it follows that $h_{k}^{D}\left(u_{N}, \Gamma\right)=1$ if $k=r(D)$ and $h_{k}^{D}\left(u_{N}, \Gamma\right)=0$ for all $k \neq r(D)$. It then follows from Lemma ?? that $\mu^{c}$ satisfies communication fairness.

5. Consider $\left(u_{T}, \Gamma\right) \in \mathcal{G}_{N}^{\Gamma_{c f}}$ and $i \in H(T) \backslash T$. Since $\Gamma$ is connected and cycle-free, by definition of hierarchical outcome it follows for every $D \in T^{s}(\Gamma)$ that $h_{k}^{D}\left(u_{T}, \Gamma\right)=1$ if and only if $k \in H(T)$ and $H(T) \subseteq \widehat{S}_{D}(k)$, and $h_{k}^{D}\left(u_{T}, \Gamma\right)=0$ otherwise. Since $i \in H(T)$, it follows that $H(T \cup\{i\})=H(T)$. Hence for every $D \in T^{s}(\Gamma)$ it follows that $h^{D}\left(u_{T \cup\{i\}}, \Gamma\right)=h^{D}\left(u_{T}, \Gamma\right)$, which shows that inside neighbor necessity is satisfied. ${ }^{3}$

6. Consider connected $T \subset N,\left(u_{T}, \Gamma\right) \in \mathcal{G}_{N}^{\Gamma_{c f}}$, and $\{i, j\} \in \Gamma$ such that $j \in T$, $i \in N \backslash T$, and $\{i, j\} \in \Gamma$. Then for every $D \in T^{s}(N, \Gamma)$ we have that either $(j, i) \in D$ or $(i, j) \in D$. In the first case we have that $h^{D}\left(u_{T \cup\{i\}}, \Gamma\right)=h^{D}\left(u_{T}, \Gamma\right)$. In the second case $h_{k}^{D}\left(u_{T \cup\{i\}}, \Gamma\right)=h_{k}^{D}\left(u_{T}, \Gamma\right)=0$ for all $k \in T \backslash\{j\} .{ }^{4}$ Hence, independence of joining outside neighbors is satisfied.

From Propositions ?? and ?? the following theorem immediately follows.

Theorem 5.1 On the class of connected cycle-free graph games the centrality rewarding Myerson value is the unique solution that satisfies efficiency, linearity, the inessential player property, communication fairness, inside neighbor necessity, and independence of joining outside neighbors.

It remains to show the logical independence of the axioms in Theorem ??.

1. The solution $f$ on $\mathcal{G}_{N}^{\Gamma_{c f}}$ given by $f(v, \Gamma)=2 \mu^{c}(v, \Gamma)$ satisfies linearity, inessential player property, communication fairness, inside neighbor necessity, and independence of joining outside neighbors. It does not satisfy efficiency.

2. Let $f$ on $\mathcal{G}_{N}^{\Gamma_{c f}}$ be given by $f(v, \Gamma)=\mu^{c}(v, \Gamma)$ when $\lambda_{T}(v)$ is not equal to zero for at most one non-empty coalition $T \subseteq N$, otherwise $f(v, \Gamma)=A T(v, \Gamma)$. Then $f$ satisfies efficiency, inessential player property, communication fairness, inside neighbor necessity, and independence of joining outside neighbors. It does not satisfy linearity.

3. Let $f$ on $\mathcal{G}_{N}^{\Gamma_{c f}}$ be given by $f_{k}(v, \Gamma)=\frac{c_{k}(\Gamma)}{\left|\Pi_{c}^{\Gamma}(N)\right|} v(N), k \in N$. Then $f$ satisfies efficiency, linearity, communication fairness, inside neighbor necessity, and independence of joining outside neighbors. It does not satisfy inessential player property.

\footnotetext{
${ }^{3}$ Note that this holds for every $i \in H(T) \backslash T$. In the axiom it is only required when $i$ is a neighbor of a player $j \in T$.

${ }^{4}$ In this case $h_{i}^{D}\left(u_{T \cup\{i\}}, \Gamma\right)=h_{j}^{D}\left(u_{T}, \Gamma\right)=1$ and $h_{i}^{D}\left(u_{T}, \Gamma\right)=h_{j}^{D}\left(u_{T \cup\{i\}}, \Gamma\right)=0$, i.e. the payoff moves from $j$ to $i$ when $T$ is replaced by $T \cup\{i\}$.
} 
4. The average tree solution $A T$ on $\mathcal{G}_{N}^{\Gamma_{c f}}$ satisfies efficiency, linearity, inessential player property, inside neighbor necessity, and independence of joining outside neighbors. It does not satisfy communication fairness.

5. Let $f$ on $\mathcal{G}_{N}^{\Gamma_{c f}}$ be given by

$$
f(v, \Gamma)=\frac{1}{\left|\Pi_{c}^{\Gamma}(N)\right|} \sum_{\pi \in \Pi_{c}^{\Gamma}(N)} m^{\pi}(v), \quad(v, \Gamma) \in \mathcal{G}_{c f}^{\Gamma},
$$

i.e. we take the average over all connectivity orderings of the marginal vectors of the game $v$ instead of the Myerson restricted game. Then $f$ satisfies efficiency, linearity, inessential player property, communication fairness, and independence of joining outside neighbors. It does not satisfy inside neighbor necessity.

6. Let $f$ be the weighted Shapley value of the Myerson restricted game with weights $\omega_{k}=\frac{c_{k}(\Gamma)}{\left|\Pi_{c}^{\Gamma}(N)\right|}, k \in N$. Then $f$ satisfies efficiency, linearity, inessential player property, communication fairness, and inside neighbor necessity. It does not satisfy independence of joining outside neighbors.

\subsection{The centrality rewarding Shapley value}

Again we consider the subclass $\mathcal{G}_{N}^{\Gamma_{c f}}$ of connected, cycle-free graph games. Since $S h^{c}$ is the average of a collection of marginal vectors of the game $v$, it follows straightforwardly that it is efficient and linear, and also satisfies the null player property. The latter property implies that it also satisfies the weaker inessential player property, but it does not satisfy the inside neighbor property because in a unanimity game $\left(u_{S}, \Gamma\right)$ an inside neighbor of $S$ may not get the same payoff as the members of $S$. Since $S h^{c}$ coincides with $\mu^{c}$ on $\left(u_{N}, \Gamma\right)$, it follows immediately that also communication fairness is satisfied. Further, from the second statement in Remark ??, in particular formula (??), it follows that $S h^{c}$ also satisfies independence of joining outside neighbors. To characterize the centrality rewarding Shapley value we replace the inside neighbor property by a new (algorithmic type) axiom. This new axiom also implies the null player property and independence of joining outside neighbors.

Without restrictions on cooperation all players in the unanimity game $u_{N}$ are symmetric. So it is reasonable that in such a case the classical Shapley value assigns equal payoffs to all players. But when cooperation is limited, the restrictions introduce asymmetries between the players and dividing the worth equally is not a priori reasonable. For instance, it might be natural to expect that a player with more connections in the communication structure is rewarded better. This basic requirement is reflected by the communication fairness axiom. When instead of the unanimity game $u_{N}$ on the grand coalition the unanimity game $u_{S}$ on a proper coalition $S \subset N$ is considered, the players outside $S$ become null-players and therefore they get a zero payoff when applying $S h^{c}$. Because of efficiency this implies that all payoffs that the players outside $S$ receive in graph game $\left(u_{N}, \Gamma\right)$ need to be reallocated among players belonging to $S$. We assume that in this redistribution procedure (i) every player is myopic; (ii) every player knows only if he belongs to $S$ or not, i.e., if he is a null-player or not, and (iii) every player knows who are his neighbors in the communication graph $\Gamma$. However, a player has 
no information whether or not his neighbors belong to $S$, and also he knows nothing about non-neighboring players. Moreover, the payoffs that players outside $S$ receive in $\left(u_{N}, \Gamma\right)$ are distributed independently of each other. Finally, we require that the payoff $f_{k}\left(u_{N}, \Gamma\right)$ of a player $k$ outside $S$ is reallocated to his neighbors proportionally to their communication abilities given in terms of the numbers of players in the components they represent in the subgraph $\left.\Gamma\right|_{N \backslash\{k\}}$, so each neighbor represents the players in the corresponding satellite. Then each neighbor of $k$ that belongs to $S$ absorbs fully his share, while a neighbor that is also outside $S$ distributes the obtained share again, now among the neighbors of himself and the null-player who initiated this redistribution step. This continues until all payoffs that the players outside $S$ receive in $u_{N}$ are reallocated to the players in $S$. It is assumed that each player has full memory concerning the origin of his possession, more precisely, he knows which part of his possession is his own share in $u_{N}$, which parts he got as the result of the reallocation of null-players possessions, and whom of the null-players was involved in the creation of this or another part of his possession. So, the described redistribution procedure is well defined and can be performed by the algorithmic procedure given below for every unanimity graph game $\left(u_{S}, \Gamma\right) \in \mathcal{G}_{N}^{\Gamma_{c f}}, S \subseteq N$, and every solution $\xi$ on the class of connected cycle-free graph games.

We first introduce some additional notation. Let $\Pi$ be a collection of linear orderings on subsets of $N$, so $\Pi$ may contain orderings of different length and on different sets of players. In the procedure below the set $\Pi$ is initiated as a set of orderings of length one. For $\pi=\left(\pi_{1}, \ldots, \pi_{r}\right) \in \Pi$, we denote the set $\left\{\pi_{1}, \ldots, \pi_{r}\right\}$ of players in $\pi$ by $N(\pi)$, and given a player $h \in N \backslash N(\pi)$, we define an ordering $\pi^{+h} \in \Pi$ as $\pi^{+h}=\left(\pi_{1}, \ldots, \pi_{r}, h\right)$. Further, for simplicity of notation, the set $N_{N(\pi)}^{\Gamma}$ of neighbors of $N(\pi)$ in $\Gamma$ is denoted by $N^{\Gamma}(\pi)$. Finally, for $h \in N^{\Gamma}(\pi)$, let $C_{h}(\pi)$ be the component $C \in\left(N \backslash N^{\Gamma}(\pi)\right) / \Gamma$ such that $C \ni h$, i.e. $C_{h}(\pi)$ contains all players outside $N^{\Gamma}(\pi)$ that are connected with $N^{\Gamma}(\pi)$ through node $h$, including $h$ itself. Given a solution $\xi$ on $\mathcal{G}_{N}^{\Gamma_{c f}}$, we then have the following algorithm to find $\xi\left(u_{S}, \Gamma\right)$ through Full Memory Myopic Proportional reallocation of $\xi\left(u_{N}, \Gamma\right)$.

\section{FMMP-Algorithm}

Step 0 Take $S \subsetneq N$. Set $x_{i}=\xi_{i}\left(u_{N}, \Gamma\right)$ for every $i \in S$ and set $\Pi=\{(i) \mid i \in N \backslash S\}$. For every $\pi=(i) \in \Pi$, set $x_{i}^{\pi}=\xi_{i}\left(u_{N}, \Gamma\right)$. Go to Step 1 .

Step 1 Take some $\pi=\left(\pi_{1}, \ldots, \pi_{r}\right) \in \Pi$. For every $h \in N^{\Gamma}(\pi)$, set $x_{h}^{\pi^{+h}}=\frac{\left|C_{h}(\pi)\right|}{|N|-\left|N^{\Gamma}(\pi)\right|} x_{\pi_{r}}^{\pi}$. Set $\Pi=\Pi \backslash\{\pi\}$. Go to Step 2 .

Step 2 For every $h \in N^{\Gamma}(\pi)$, set $x_{h}=x_{h}+x_{h}^{\pi^{+h}}$ if $h \in S$ and set $\Pi=\Pi \cup\left\{\pi^{+h}\right\}$ if $h \notin S$. Go to Step 3 .

Step 3 If $|\Pi|>0$, return to Step 1. Otherwise, set $\xi_{i}\left(u_{S}, \Gamma\right)=x_{i}$ if $i \in S$ and $\xi_{i}\left(u_{S}, \Gamma\right)=0$ otherwise. Stop.

We now state the following axiom. 


\section{Full Memory Myopic Proportional Fairness}

For any $\left(u_{T}, \Gamma\right) \in \mathcal{G}_{N}^{\Gamma_{c f}}, T \subset N$, it holds that $f\left(u_{T}, \Gamma\right)$ is obtained from $f\left(u_{N}, \Gamma\right)$ by applying the FMMP-algorithm.

Theorem 5.2 On the class of connected cycle-free graph games the centrality rewarding Shapley value is the unique solution that satisfies efficiency, linearity, communication fairness, and full memory myopic proportional fairness.

\section{Proof.}

For every connected cycle-free graph $\Gamma$, the payoff vector $f\left(u_{N}, \Gamma\right)$ is uniquely determined by efficiency and communication fairness. Then for every $T \subset N, f\left(u_{T}, \Gamma\right)$ follows from the full memory myopic proportional fairness. By linearity $f(v, \Gamma)$ is uniquely determined for any $(v, \Gamma) \in \mathcal{G}_{N}^{\Gamma_{c f}}$.

To show existence, note that $S h^{c}$ satisfies efficiency and linearity. Since it coincides with $\mu^{c}$ when $v=u_{N}$ it also satisfies communication fairness. For $T \subset N$, the FMMP algorithm distributes for every $k$ outside $T$ its payoff $S h_{k}^{c}\left(u_{N}, \Gamma\right)$ in the unanimity game on the grand coalition to the border nodes of $T$. The FMMP algorithm gives each $i \in T$ payoff

$c_{i}(\Gamma)+\sum_{C \in((N \backslash S) / \Gamma)^{i}} \sum_{j \in C} \sum_{Q \in C_{i}^{\Gamma}(C): Q \ni j} c_{j}\left(\left.\Gamma\right|_{Q}\right) \cdot\left(\begin{array}{c}|N \backslash Q|-1 \\ |K|, K \in\left(N \backslash Q_{+i}\right) / \Gamma\end{array}\right) \prod_{h \in N_{Q_{+i}}^{\Gamma}} c_{h}\left(\left.\Gamma\right|_{\left(\left(N \backslash Q_{+i}\right) / \Gamma\right)_{h}}\right)$.

Changing the order of the second and third summation in the second term yields formula (??). This shows that $S h^{c}$ satisfies full memory myopic proportional fairness.

It remains to show the logical independence of the four axioms in Theorem ??.

1. The solution $f$ on $\mathcal{G}_{N}^{\Gamma_{c f}}$ given by $f(v, \Gamma)=2 S h^{c}(v, \Gamma)$ satisfies linearity, communication fairness, and full memory myopic proportional fairness. It does not satisfy efficiency.

2. Let $f$ on $\mathcal{G}_{N}^{\Gamma_{c f}}$ be given by $f(v, \Gamma)=S h^{c}(v, \Gamma)$ when $\lambda_{T}(V)$ is not equal to zero for at most one non-empty coalition $T \subseteq N$, otherwise $f(v, \Gamma)=A T(v, \Gamma)$. Then $f$ satisfies efficiency, communication fairness, and full memory myopic proportional fairness. It does not satisfy linearity.

3. Let $f$ on $\mathcal{G}_{N}^{\Gamma_{c f}}$ be given by (i) $f\left(u_{N}, \Gamma\right)=A T\left(u_{N}, \Gamma\right)$, (ii) for every $S \subset N$ the payoff vector $f\left(u_{S}, \Gamma\right)$ is obtained by applying the FMMP algorithm and (iii) $f(v, \Gamma)=\sum_{S \subseteq N, S \neq \emptyset} \lambda_{S}(v) f\left(u_{S}, \Gamma\right)$. Then $f$ satisfies efficiency, linearity, and full memory myopic proportional fairness. It does not satisfy communication fairness.

4. Let $f$ on $\mathcal{G}_{N}^{\Gamma_{c f}}$ be given by $f_{k}(v, \Gamma)=\frac{c_{k}(\Gamma)}{\mid \Pi_{c}^{\Gamma}(N)} v(N), k \in N$. Then $f$ satisfies efficiency, linearity, and communication fairness. It does not satisfy full memory myopic proportional fairness. 


\section{$6 \quad$ Further remarks}

In this paper we introduce two solutions for graph games that rewards centrality. The centrality rewarding Myerson value rewards also for communication necessity, whereas the centrality rewarding Shapley value has the null player property. As shown in Section 3 existing values for graph games as the Myerson value and the average tree solution reward for communication necessity, but not for centrality in the graph, while the solution of Bilbao and the gravity center punish for centrality. Note from the examples showing the logical independence that the average tree solution satisfies all the axioms of Theorem ??, except communication fairness. Instead the average tree solution is characterized by the symmetry axiom saying that, $f_{i}\left(c u_{N}, \Gamma\right)=f_{j}\left(c u_{N}, \Gamma\right)$ for all $i, j \in N$ and real numbers $c$, see Mishra and Talman (2010). This axiom requires that every player gets the same payoff when the game is (a multiple of) the unanimity game on the grand coalition, irrespective of the underlying graph. The Myerson value also satisfies this symmetry requirement together with efficiency, linearity, inessential player property, and inside neighbor necessity, but it does not satisfy independence of joining outside neighbors.

Another class of values that rewards for communication and centrality is the class of Harsanyi power solutions introduced in van den Brink, van der Laan. and Pruzhansky (2011). A power (or centrality) measure answers the question which nodes in a graph under consideration are important. In fact, it gives a complete or partial ordering of the nodes with respect to importance, cohesiveness, or influence. On the class $\Gamma_{N}^{c}$ of connected graphs, a power measure is a function $p$ which assigns to each graph $\Gamma \in \Gamma_{N}^{c}$ a non-zero vector $p(\Gamma) \in \mathbb{R}_{+}^{N}$. The entry $p_{i}(\Gamma)$ measures the power (or centrality) of node $i$ in graph $\Gamma$, the higher $p_{i}(\Gamma)$ is, the higher the influence of node $i$ within the graph. A well-known power measure is the degree measure which assigns to any graph the vector of degrees of its nodes. For a graph $\Gamma$ and connected set $S \subseteq N$, the vector $p\left(\left.\Gamma\right|_{S}\right) \in \mathbb{R}_{+}^{S}$ measures the power of the nodes of $S$ in the subgraph of $\Gamma$ on $S$. Given a power function $p$, the Harsanyi power solution $f^{p}$ on the class of connected graph games assigns to every $(v, \Gamma) \in \Gamma_{c}^{N}$ the vector $f^{p}(v, \Gamma) \in \mathbb{R}^{N}$ given by

$$
f_{i}^{p}(v, \Gamma)=\sum_{S \subseteq N: i \in S} p_{i}\left(\left.\Gamma\right|_{S}\right) \lambda_{v^{\Gamma}}(S), \quad i \in N,
$$

i.e. for every connected coalition $S$ the Harsany dividend of $S$ in the Myerson restricted game is distributed amongst the players in $S$ according to the power measure on the subgraph $\left.\Gamma\right|_{S}$. Since the Harsanyi power solution distributes the dividends of the Myerson restricted game, it respects communication necessity. Further, since all dividends are distributed according to the power measure, it rewards centrality as far as the power measure reflects centrality. For instance, consider a linear graph and take the degree measure. Then for any connected set $S$ the power of the two extreme nodes of $S$ in the subgraph $\left.\Gamma\right|_{S}$ is equal to one, and all other nodes have power equal to two. So, the dividend of a connected coalition $S$ in the Myerson restricted game is distributed such that the share of every non-extreme node is twice as big as the shares of the two extreme nodes of $S$. It is easy to verify that any Harsanyi power solution satisfies efficiency, linearity, inessential player property, inside neighbor necessity, and a modified symmetry axiom, namely that in a unanimity game on the grand coalition the payoff 
is distributed according to the power measure on $\Gamma$. It does not satisfy independence of joining outside neighbors. A disadvantage is that the distribution of the dividends depends on an exogenous given power function, whereas the centrality feature of the two centrality rewarding values result from the endogenous determined communication fairness. Another difference between the two centrality rewarding values and the Harsanyi power solutions is that the distribution of the dividends according to the new values depends on the full graph $\Gamma$, whereas the distribution of the dividend of a coalition $S$ according to a Harsanyi power solution only depends on the subgraph $\left.\Gamma\right|_{S}$. In fact, on the class of connected cycle-free graph games the two values reward a player in the border of $S$ for representing the players outside $S$ that are connected by that player to the other players in $S$, whereas a Harsanyi power solution ignores this.

When taking the degree measure the Harsanyi power solution is equal to the position value introduced by Borm, Owen and Tijs (1992) on the class of cycle-free graph games. This implies that also the position value reflects centrality. However, in case there exists cycles the position value is not equal to the Harsanyi (degree) power solution and the position value has the disadvantage that it might reward players that do not generate value (i.e. they are null players in the game) and not needed for communication between the non-null players. For instance, consider the graph of Example ?? and characteristic function $u_{124}$. Then player 3 is a null player in the game and is not needed for the communication between the players 1,2 and 4, but receives positive payoff $\frac{1}{6}$ according to the position value. In fact, for this example the position value gives payoff vector $\left(\frac{11}{24}, \frac{1}{6}, \frac{1}{12}, \frac{7}{24}\right)$ and the Harsanyi (degree) power solution gives payoff vector $\left(\frac{1}{2}, \frac{1}{4}, 0, \frac{1}{4}\right)$. Recall that in Example ?? we found that the centrality rewarding value gives payoff vector $\left(\frac{8}{14}, \frac{4}{14}, 0, \frac{2}{14}\right)$.

According to equation (??) the centrality rewarding Myerson value assigns to any connected cycle-free graph game the weighted average of hierarchical outcomes with weights the fractions of connectivity orderings having each player as last player. For an exogenous vector of weights $\omega \in \mathbb{R}_{+}^{N}$, with sum of the weights equal to one, on the class of connected cycle-free graph games we can define the value $\mu^{\omega}$ as the solution that assigns to every $(v, \Gamma) \in \mathcal{G}_{N}^{\Gamma_{c f}}$ the weighted average of the hierarchical outcomes given by

$$
\mu^{\omega}(v, \Gamma)=\sum_{i \in N} \omega_{i} h^{D_{i}}(v, \Gamma)
$$

It follows that $\mu^{c}(v, \Gamma)=\mu^{\omega}(v, \Gamma)$ when $\omega_{i}=\frac{c_{i}(\Gamma)}{\mid \Pi_{c}^{\Gamma}(N)}, i \in N$ and that $A T(v, \Gamma)=$ $\mu^{\omega}(N, v, \Gamma)$ with $\omega_{i}=\frac{1}{n}$ for all $i \in N$. So, on the class of connected cycle-free graph games the only difference between $\mu^{c}$ and the $A T$ solution is the vector of weights. It is straightforward to verify that for given weight vector $\omega \in \mathbb{R}_{+}^{N}$ (with all weights strictly positive and sum of weights equal to one), the solution $\mu^{\omega}$ on the class of connected cyclefree graph games is characterized by efficiency, linearity, the inessential player property, inside neighbor necessity, independence of joining outside neighbors, and the weighted symmetry axiom saying that $f_{i}\left(u_{N}, \Gamma\right)=\omega_{i}$ for all $i \in N .^{5}$ Indeed, the weighted symmetry axiom coincides with the communication fairness axiom when $\omega_{i}=\frac{c_{i}(\Gamma)}{\mid \Pi_{c}^{\Gamma}(N)}, i \in N$. Again, the latter axiom is induced endogenously by the graph, whereas in the weighted

\footnotetext{
${ }^{5}$ In van den Brink et al. (2011) this axiom is called $\omega$-communication ability on the class of graph games.
} 
symmetry axiom the weights are given exogenously. Also note that any (exogneous given) power function (or centrality measure) $p$ induces weights $\omega_{i}=\frac{p_{i}(\Gamma)}{\sum_{k \in N} p_{k}(\Gamma)}$ for all $i \in N$, and therefore yields a corresponding solution $\mu^{\omega}$. Finally, we remark that within the framework of river games the value $\mu^{\omega}$ has been characterized on the class $\mathcal{G}_{N}^{\Gamma_{c f}}$ in van den Brink, van der Laan, and Moes (2012) as the unique solution that satisfies efficiency and $\omega$-fairness ${ }^{6}$, where a solution $f$ satisfies $\omega$-fairness when for every $(v, \Gamma) \in \mathcal{G}_{N}^{\Gamma_{c f}}$ and $\{k, h\} \in \Gamma$ it holds that

$$
\frac{\sum_{i \in N_{h}(\Gamma \backslash\{k, h\})} f_{i}(v, \Gamma)-v\left(N_{h}(\Gamma \backslash\{k, h\})\right.}{\sum_{i \in N_{k}(\Gamma \backslash\{k, h\})} f_{i}(v, \Gamma)-v\left(N_{k}(\Gamma \backslash\{k, h\})\right.}=\frac{\sum_{i \in N_{h}(\Gamma \backslash\{k, h\})} \omega_{i}}{\sum_{i \in N_{k}(\Gamma \backslash\{k, h\})} \omega_{i}} .
$$

It means that after deleting a link the ratio of the total payoffs in the two resulting components minus the own worth of the components is equal to the ratio of the total weights in these two components. In case that all weights are equal, this reduces to component fairness introduced in Herings et al. (2008) for characterizing the average tree solution.

\section{References}

[1] Bilbao, J.M. (2003), Cooperative games under augmenting systems, SIAM Journal of Discrete Mathematics, 17, 123-133.

[2] Borm P., G. Owen, and S. Tijs (1992), On the position value for communication situations, SIAM Journal on Discrete Mathematics, 5, 305-320.

[3] Brink, J.R. van den (1999), Comparable axiomatizations of the Myerson value, the restricted Banzhaf value, hierarchical outcomes and the average tree solution for cycle-free graph restricted games, Tinbergen Discussion Paper TI 2009-108/1, Tinbergen Institute, Amsterdam, The Netherlands.

[4] Brink, J.R. van den (2012), Efficiency and collusion neutrality in cooperative games and networks, Games and Economic Behavior, 76, 344-348.

[5] Brink, J.R. van den, C. Dietz, G. van der Laan, and G. Xu (2016), Comparable characterizations of four solutions for permission tree games, forthcoming in Economic Theory.

[6] Brink, J.R. van den, P.J.J. Herings, G. van der Laan, and A.J.J. Talman (2015), The average tree permission value for games with a permission tree, Economic Theory, $58,99-123$.

[7] Brink, J.R. van den, G. van der Laan, and N. Moes (2012), Fair agreements for sharing international rivers with multiple springs and externalities games, Journal of Environmental Economics and Management, 63, 388-403.

\footnotetext{
${ }^{6}$ Actually it is called $\omega$-TIBS fairness, where TIBS refers to a principle of fairness in International Water Law.
} 
[8] Brink, J.R. van den, G. van der Laan, and V. Pruzhansky (2011), Harsanyi power solutions for graph-restricted games, International Journal of Game Theory, 40, $87-110$.

[9] Demange, G. (2004), On group stability in hierarchies and networks, Journal of Political Economy 112, 754-778.

[10] Haller, H. (1994), Collusion properties of values, International Journal of Game Theory, 23, 261-281.

[11] Harsanyi, J.C. (1959), A bargaining model for cooperative $n$-person games, in: Tucker, A.W., and R.D Luce (eds.) Contributions to the theory of games IV, Princeton University Press, Princeton NJ, pp. 325-355.

[12] Herings, P.J.J., G. van der Laan, and A.J.J. Talman (2008), The average tree solution for cycle-free graph games, Games and Economic Behavior 62, 77-92.

[13] Herings, P.J.J., G. van der Laan, A.J.J. Talman, and Z. Yang (2010), The average tree solution for cooperative games with communication structure, Games and Economic Behavior 68, 626-633.

[14] Khmelnitskaya, A.B., G. van der Laan, and A.J.J. Talman (2016), Generalization of binomial coefficients to numbers on the nodes of graphs, Tinbergen Discussion Paper TI 2016-011/II, Tinbergen Institute, Amsterdam, The Netherlands.

[15] Koshevoy, G.A. and A.J.J. Talman (2014), Solution concepts for games with general coalitional structure, Mathematical Social Sciences 68, 19-30.

[16] Malawski, M. (2002), Equal treatment, symmetry and Banzhaf value axiomatizations, International Journal of Game Theory, 31, 47-67.

[17] Mishra, D. and A.J.J. Talman (2010), A characterization of the average tree solution for tree games, International Journal of Game Theory, 39, 105-111.

[18] Myerson, R.B. (1977), Graphs and cooperation in games, Mathematics of Operations Research, 2, 225-229.

[19] Shapley, L.S. (1953), A value for n-person games, in: Kuhn, H.W., and A.W. Tucker (eds.), Contributions to the Theory of Games II, Princeton University Press, Princeton NJ, pp. 307-317.

[20] Suzuki, T. (2015), Solutions for Cooperative Games with and without Transferable Utility, PH.D Thesis, Center for Economic Research, Tilburg University, The Netherlands. 